Institute of Medical Physics and Biophysics

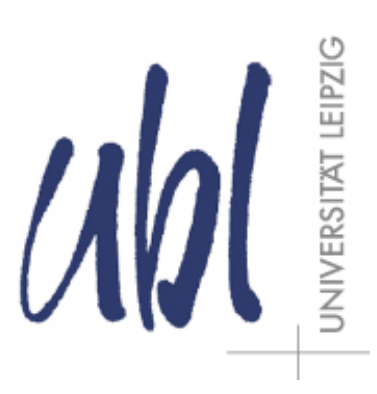

Leipzig University

Author Manuscript

Published in final edited form as:

Biochim. Biophys. Acta (Molecular and Cell Biology of

Lipids), 2014 Aug; 1841(8):1146-1160.

DOI: $10.1016 /$ ibbalip.2013.12.002

(C) 2014. This manuscript version is made available

under the CC-BY-NC-ND 4.0 licence

http://creativecommons.org/licenses/by-nc-nd/4.0/

\title{
SOLID-STATE NMR SPECTROSCOPY TO STUDY PROTEIN-LIPID INTERACTIONS
}

\author{
Daniel Huster
}

Institute of Medical Physics and Biophysics, University of Leipzig, Härtelstr. 16 -

18, D-04107 Leipzig, Germany

\begin{abstract}
The appropriate lipid environment is crucial for the proper function of membrane proteins. There is a tremendous variety of lipid molecules in the membrane and so far it is often unclear which component of the lipid matrix is essential for the function of a respective protein. Lipid molecules and proteins mutually influence each other; parameters such as acyl chain order, membrane thickness, membrane elasticity, permeability, lipid-domain and annulus formation are strongly modulated by proteins. More recent data also indicates that the influence of proteins goes beyond a single annulus of next-neighbor boundary lipids. Therefore, a mesoscopic approach to membrane lipid-protein interactions in terms of elastic membrane deformations has been developed. Solid-state NMR has greatly contributed to the understanding of lipid-protein interactions and the modern view of biological membranes. Methods that detect the influence of proteins on the membrane as well as direct lipid-protein interactions have been developed and are reviewed here. Examples for solid-state NMR studies on the interaction of Ras proteins, the antimicrobial peptide protegrin-1, the $\mathrm{G}$ protein-coupled receptor rhodopsin, and the $\mathrm{K}^{+}$channel $\mathrm{KcsA}$ are discussed.
\end{abstract}

\section{Abbreviations}

AFM, atomic force microscopy; CP, cross-polarization; CSA, chemical shift anisotropy; DHA, docosahexaenoic acid; GPCR, G protein-coupled receptor; MAS, magic-angle spinning; NOE, nuclear Overhauser effect; NOESY; nuclear Overhauser enhancement spectroscopy; PC, phosphatidylcholine, PE, phosphatidylethanolamine; PG, phosphatidylglycerol; PFG, pulsed field gradients; ROS, rod outer segment; REDOR, rotational-echo double resonance; STD, saturation transfer difference 


\section{Introduction}

The biological membrane represents the most important and most highly specialized interface in the human body. All essential membrane properties are caused by a complex interplay between lipid molecules and the proteins embedded or associated with the lipid membrane. Membrane proteins influence the structure and dynamics of the surrounding lipids [1]. Typical membrane parameters that are influenced by proteins are acyl chain order, membrane thickness, membrane elasticity, permeability, lipid-domain and annulus formation, headgroup orientation, and acyl chain dynamics [2]. In turn, the lipid membrane has a profound impact on the structure and dynamics of membrane embedded and membrane-associated proteins and peptides [3] and can directly interfere with the function of these molecules $[2,4,5]$.

The lipid environment of a protein represents a large challenge and the understanding, which component of the lipid membrane is crucial for protein function is fragmentary. It still remains an unsolved problem why different cellular membranes show quite a variety in lipid composition and why the putatively basic function of the phospholipid bilayer, which is to provide the 'solvent' for the membrane proteins [6] requires such a tremendous variety of lipid species. However, the original view of the membrane, where the lipid bilayer represents the structural scaffold and that lipids and proteins represent a fluid mosaic [6] has been modified since this model was first developed in 1972. Especially the raft hypothesis [7], formulated in 1997, and the discovery of lipid molecules acting as second messengers $[8,9]$ has highlighted the functional importance of the lipids of a biological membrane. It was originally formulated in the fluid mosaic model that "the phospholipids and proteins of membranes do not interact strongly; in fact, they appear to be largely independent" [6]. But now it is clear that membrane lipids strongly affect proteins and vice versa. Crystal structures of many membrane proteins show tightly bound non-annular lipids [10]. For instance, the crystal structure of the $\mathrm{K}^{+}$channel KcsA shows two lipid molecules modeled as nonan-1-ol and diacylglycerol [11]. Furthermore, cholesterol has been identified in crystal structures of $\mathrm{G}$ protein-coupled receptors [12] and was also shown to bind the flexible transmembrane domain of the amyloid precursor protein [13].

The hydrophobic surface of a membrane protein is heterogeneous and rough and covered by a shell of annual lipids acting as solvent for the protein [10].The lipids in this shell, which directly interact with the protein, experience essential physical interactions such as hydrogen bonds, $\pi-\pi$ and cation- $\pi$ interactions, electrostatic and van der Waals forces [14]. But the mutual influence of lipids on proteins goes beyond a single annulus of nextneighbor boundary lipids [15]. It is well known that the chemical/physical nature of the lipid membrane can strongly modulate the function of membrane proteins $[2,4,5]$. Thus, membrane curvature forces [15] and hydrophobic mismatch [16] represent concepts that consider a mesoscopic approach to membrane lipid-protein interactions in terms of elastic membrane deformations [14].

Both mechanisms of lipid-protein interaction, either via specific interactions with the lipids of the first layer or elastic membrane deformations, can be studied by solid-state NMR spectroscopy. In Fig. 1, a schematic overview of the parameters relevant for lipid-protein interaction that can be studied by solid-state NMR is given. 
Preparations of membrane reconstituted proteins represent challenging NMR samples because of the high degree of molecular dynamics. The lipids in membranes in the liquid crystalline phase are highly dynamic and show motions on several time scales, including large amplitude motions, which sufficiently scale down anisotropic NMR interactions such that even well-resolved ${ }^{1} \mathrm{H}$ NMR spectra can be recorded under magic-angle spinning (MAS) conditions [17]. Membrane proteins are also dynamic molecules but timescales and geometry of these motions are typically not compliant with high resolution MAS NMR. To study membrane proteins, usually ${ }^{13} \mathrm{C}$ or ${ }^{15} \mathrm{~N}$ cross-polarization MAS NMR methods under high power decoupling are employed [18]. Thus, studying lipid-protein interactions by solidstate NMR utilizes both approaches and methodological development has to adapt the technique to the specificity of the interface between highly mobile lipids and less mobile proteins.

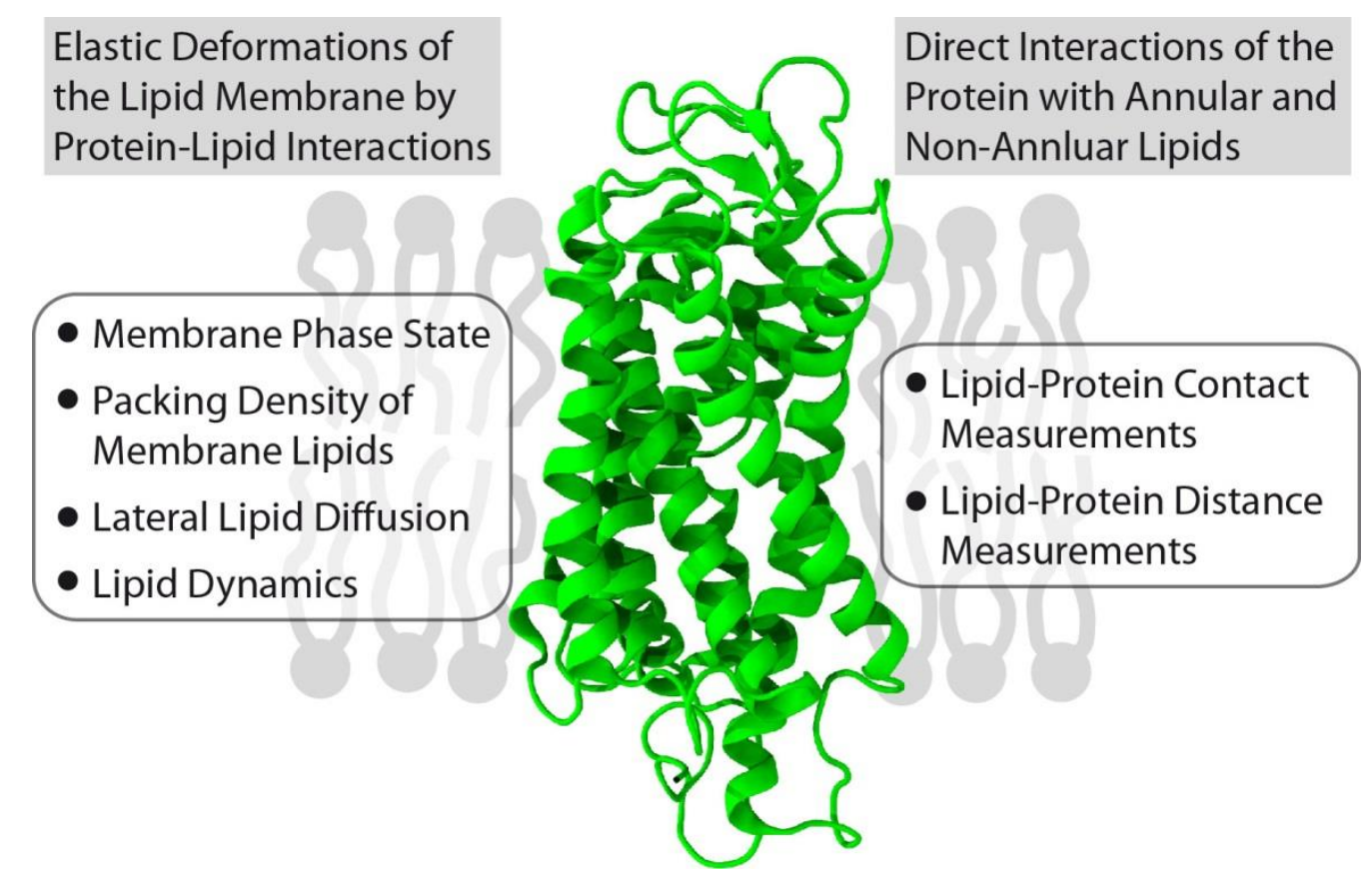

Figure 1. Schematic overview of the two principle approaches to study lipid-protein interactions in membranes by solid-state NMR spectroscopy. Key properties that can be probed are exemplified.

In what follows, a short overview over the solid-state NMR technique will be given. Then, the individual methods developed to study the influence of proteins on the lipid membrane and direct lipid-protein contacts are introduced. We continue with examples from the recent literature that summarize the capability of the method, before we conclude with some limitations of the current technology. Overall, solid-state NMR spectroscopy has continuously developed for more than 40 years and established itself as an indispensable tool to study lipid-protein interactions in many fields of biological sciences.

\section{Solid-State NMR Methodology}

\section{1. Short Introduction into Solid-State NMR Spectroscopy of Lipid Membranes and Membrane Proteins}

Biological membranes are complex interfaces of restricted geometry and constraint mobility of the molecules forming the membrane. The building principle of these structures 
is the hydrophobic effect [19], which results in the clustering of hydrophobic segments of lipids and proteins due to the release of water from these molecular moieties, which increases the water entropy and decreases the free energy of the assembly. This creates the typical bilayer structure, where lipid headgroups and polar protein segments face the aqueous phases on either side of the membrane and the hydrophobic moieties of the membrane forming molecules build the membrane interior. It should be emphasized, however, that this assembly is highly mobile and the degree of molecular order especially in the lipid phase is very low. Intense research over the last 15 years has revealed the high degree of molecular dynamics and disorder of lipid membranes, in particular using diffraction techniques [1,20], solid-state NMR spectroscopy [21-24], and molecular dynamics simulations $[20,25,26]$. The molecular dynamics of the membrane comprises fast segmental motions on the picosecond time scale $[25,27]$, whole body lipid rotations and tilting on the nanosecond time scale $[28,29]$, and lateral diffusion of the lipids with diffusion coefficients on the order of $10^{-10}-10^{-12} \mathrm{~m}^{2} / \mathrm{s}$ [30]. Also membrane proteins [31-34] and membrane-associated proteins $[35,36]$ are recognized as highly mobile molecules. Protein motions include fast segmental motions on the nanosecond time scale, rotational and tilting motions of entire membrane proteins on the microsecond time scale, and lateral diffusion featuring diffusion coefficients on the order $10^{-12} \mathrm{~m}^{2} / \mathrm{s}$ and larger [37].

In spite of this comprehensive molecular dynamics, from the NMR point of view, biological and model membranes are solids and consequently do not provide highly resolved NMR spectra. While in solution, rapid isotropic motions with correlation times of a few nanoseconds average anisotropic interactions to zero, the molecular motions in membranes are anisotropic and substantial residual anisotropic interactions remain. This is illustrated for the NMR chemical shift, which is the most central experimental parameter determined in NMR spectroscopy. As seen from eq. (1), the expression for the NMR chemical shift has two contributions, the well-known isotropic chemical shift from solution NMR ( $\left.\omega_{\text {iso }}\right)$ and an anisotropic component relevant to all solid-state NMR techniques ( $\omega_{\text {aniso }}$ ) [38,39]:

$$
\omega=\omega_{\text {iso }}+\omega_{\text {aniso }}=\sigma_{\text {iso }} \gamma B_{0}+\frac{\delta}{2}\left(3 \cos ^{2} \theta-1-\eta \sin ^{2} \theta \cos (2 \phi)\right) .
$$

In the first term, $\sigma_{\text {iso }}$ is the isotropic chemical shift that provides a measure of the influence of the local electronic environment of a specific nucleus on the Larmor frequency. The symbols $\gamma$ and $B_{0}$ are the gyromagnetic ratio (a constant for a given nucleus) and the external magnetic field, respectively. The second term in eq. (1) considers the fact that the electronic environment of a nucleus in a given chemical structure is not isotropic, which arises from the specific structure and nature of the chemical bonds and chemical groups in the direct vicinity of the nucleus. Consequently, the screening of the external magnetic field depends on the orientation of a molecule within this field, thus, the screening of the nucleus in the anisotropic electronic environment is most conveniently described by the second rank tensor of the chemical shift [38,39]. This tensor is diagonal in the principle axis system and the angles $\theta$ and $\phi$ represent the polar angles that position the principle axis system of the chemical shift tensor in the laboratory frame, where $B_{0}$ is along the $z$ direction. The symbols $\delta$ and $\eta$ are the anisotropy parameter and asymmetry parameter of the chemical shift tensor, respectively. 


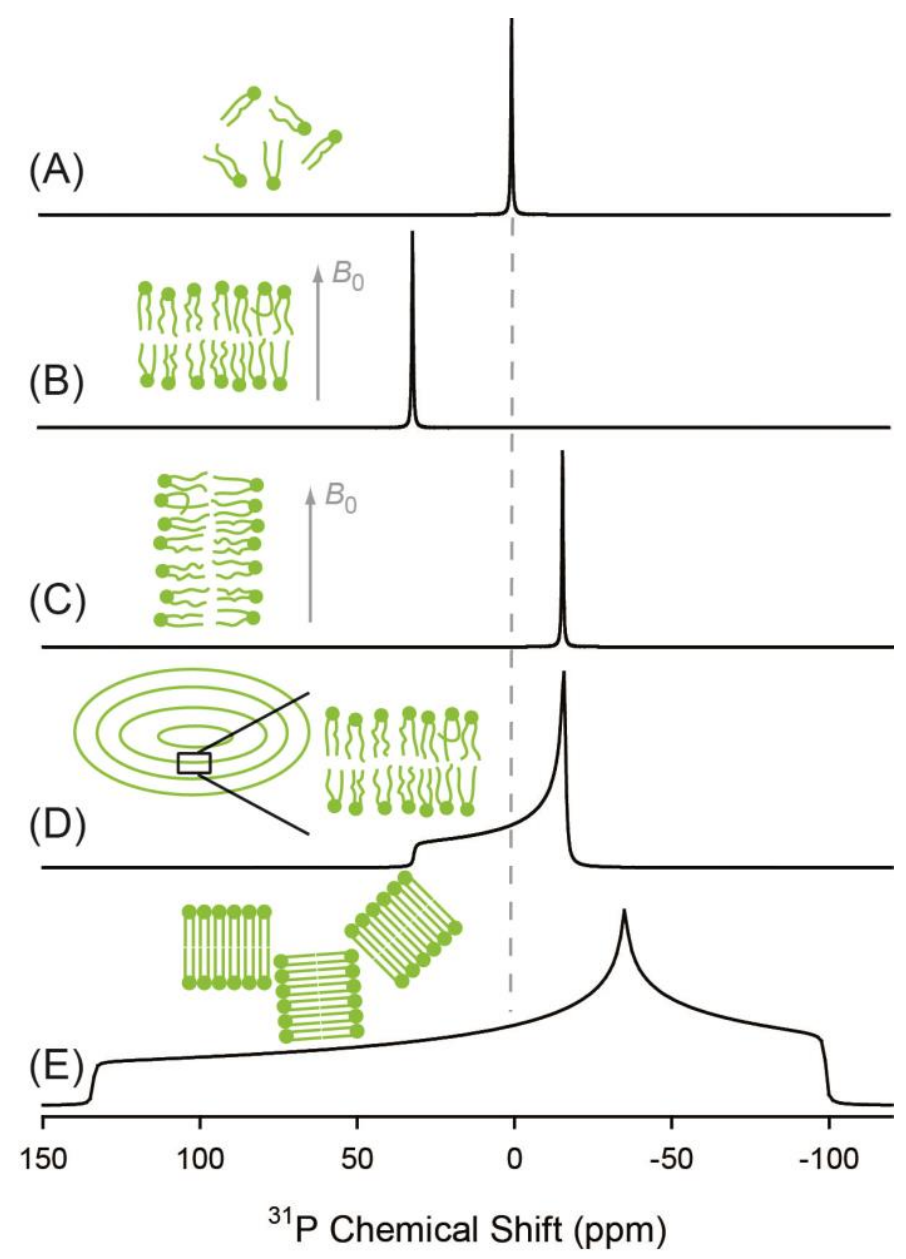

Figure 2. Simulated ${ }^{31} \mathrm{P}$ NMR spectra of phospholipids in solution, bilayers, and crystals. Isotropically mobile phospholipids in solution provide narrow ${ }^{31} \mathrm{P} N \mathrm{NM}$ lines at the isotropic chemical shift $\sigma_{\text {iso }}=2 \mathrm{ppm}$ ) of the respective lipid (A). Liquid-crystalline membranes, macroscopically oriented with the membrane normal parallel $(B)$ and antiparallel $(C)$ to the external magnetic field show narrow ${ }^{31} \mathrm{P}$ NMR lines at an anisotropic chemical shift of around $\sigma_{\text {iso }}+30 \mathrm{ppm}(\mathrm{B})$ or $\sigma_{\text {iso }}-15 \mathrm{ppm}$ (C) depending on the orientation. Phospholipids in multilamellar vesicles in the liquid-crystalline phase state show spectra with a continuous intensity distribution according to the orientation of each molecule relative to the external magnetic field according to eq. (1) (D). Note that due to the axially symmetric rotation of the molecules in the bilayer the asymmetry parameter $\sigma=0$. Crystalline phospholipids in the absence of water that undergo no molecular dynamics give rise to very broad NMR spectra representing the full width of the chemical shift anisotropy tensor $(E)$. The dotted line indicates the isotropic chemical shift for the respective lipid.

Eq. (1) illustrates the most important difference between solution and solid-state NMR spectroscopy: while the chemical shift in solution is a truly isotropic quantity, the NMR frequencies acquire a dependence on the orientation of a molecule in the magnetic field when the molecular motions are not isotropic on the fast time scale or completely absent. This shall be illustrated on the ${ }^{31} \mathrm{P}$ NMR line shapes of phospholipids in various preparations shown in Fig. 2. Phospholipid molecules in organic solvent undergo fast isotropic motions, which lead to the complete averaging of the anisotropic part of the CSA tensor and a narrow line is detected at the isotropic chemical shift of $\sigma_{\text {iso }}=2 \mathrm{ppm}(\mathrm{A})$. Next, we consider a lipid membrane in the liquid-crystalline phase state, where the molecules undergo fast axially symmetric motions leading to a partial averaging of the chemical shift anisotropy 
tensor such that the asymmetry parameter $\eta=0$ and the anisotropy parameter $\delta$ represents a motionally scaled value of $\langle\delta\rangle=30 \mathrm{ppm}$ [40]. In this situation, only the angle $\theta$ in eq. (1) has to be considered. If one prepares membranes between glass plates with the long axis of the lipids parallel to the external magnetic field, $\theta=0^{\circ}$ and a single line arises at a chemical shift of $\sigma=\sigma_{\text {iso }}+30 \mathrm{ppm}$ (B). Thus, the chemical shift now explicitly contains the orientation of the molecule in the magnetic field. If the glass plates with the oriented membranes are tilted by $\theta=90^{\circ}$ with respect to the $B_{0}$ field, eq. (1) yields a single line with a chemical shift of $\sigma=\sigma_{\text {iso }}-15 \mathrm{ppm}$ (C). Again, the NMR spectrum carries an explicit orientation dependence, although the chemical nature of the molecule investigated is exactly the same as in solution. Next, we consider a spherical phospholipid vesicle in the liquid crystalline phase state. The diameter of this vesicle is large enough so that lateral diffusion does not lead to an averaging of the anisotropic effects ( $d>100 \mathrm{~nm}$ ). In this situation, all orientations of the phospholipids are found and for the calculation of the NMR spectrum of such vesicles, all orientations, i.e. all possible $\theta$ angles have to be considered, which is done by the integration of eq. (1) according to $\int \omega(\theta) \sin \theta d \theta$ (due to the fast axially symmetric reorientation of the phospholipids, $\eta=0$ and the angle $\phi$ does not have to be considered). This leads to a broad NMR signal called powder spectrum as shown in Fig. 2D. Finally, we consider crystalline phospholipids in the absence of all motions. Under these circumstances, no motional averaging narrows the NMR powder pattern and both $\theta$ and $\phi$ angles have to be considered for the calculation of the NMR spectrum according to $\iint \omega(\theta, \phi) \sin \theta d \theta d \phi$. The resulting powder spectrum now covers a much larger spectral region and provides a distinctly different line shape (Fig. 2E). As seen from Fig 2(B-E), NMR powder spectra contain a wealth of information with regard to the structure and orientation of molecules in the magnetic field, which can be directly deduced from the spectra (vide infra).

While NMR powder spectra and anisotropic chemical shifts contain a multitude of structural and dynamical data, they also feature a significant disadvantage. Due to the large chemical shift span, NMR powder spectra occupy a significant portion of the chemical shift range and typically only the superposition a few such powder spectra can be analyzed. For larger molecules with many nuclei of the same kind, powder NMR spectra are typically not useful as they consist of a featureless broad overlay of individual powder patterns. Clearly, achieving high resolution in solid-state NMR would be very advantageous. One way to convert the broad powder spectra into relatively narrow NMR lines is the macroscopic orientation as discussed above. For studying membranes and membrane proteins, this technique indeed provides relatively narrow NMR spectra [41,42]. Individual peaks of the amides in ${ }^{15} \mathrm{~N}$ NMR spectra or individual lipid classes in the ${ }^{31} \mathrm{P} N M R$ spectra can often be resolved in that way [42]. However, it should be noted that the chemical shifts observed for oriented samples represent the anisotropic chemical shift values for a specific set of $\theta$ and $\phi$ angles according to eq. (1) in spite of the fact that narrow NMR lines are detected. These anisotropic chemical shifts do provide information about the orientation of a specific ${ }^{15} \mathrm{~N}$ amide tensor and thus about transmembrane or surface bound $\alpha$-helices, but not about the secondary structure as isotropic chemical shifts do [43]. Care should be taken when preparing oriented membrane stacks with membrane proteins and the orientation should 
always be tested in simple ${ }^{31} \mathrm{P}$ NMR experiments as it is often experimentally difficult to achieve good orientation of larger membrane proteins in such preparations.

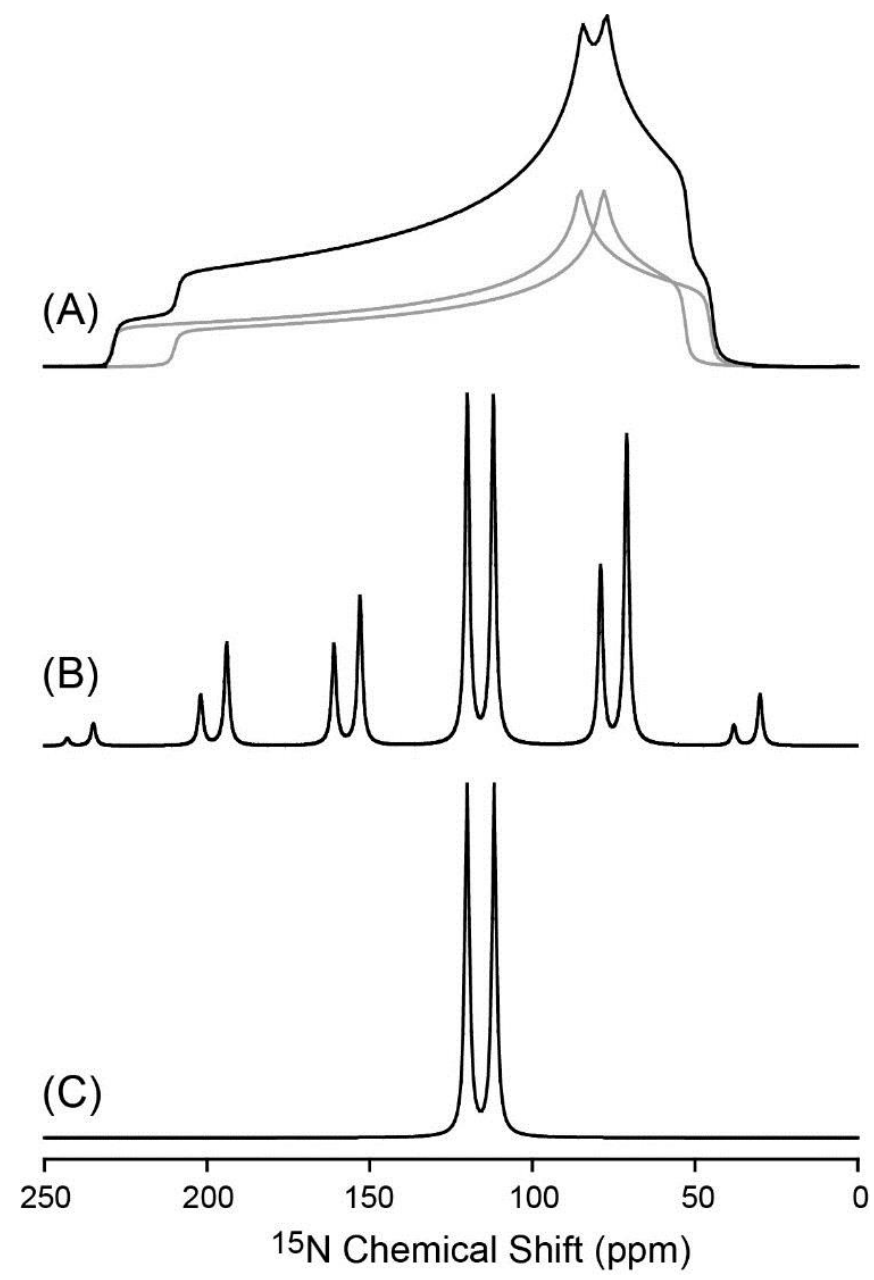

Figure 3. Simulated proton decoupled ${ }^{15} \mathrm{~N}$ solid-state NMR spectra of a membrane protein with two amino acids ${ }^{15} \mathrm{~N}$-labeld. (A) static ${ }^{15} \mathrm{~N}$ NMR spectra showing the superposition of the two anisotropic powder patterns. Magic-angle spinning with a frequency of $2.5 \mathrm{kHz}$ converts the powder pattern into the center band and a series of narrow lines (B) Fast MAS at a frequency of $10 \mathrm{kHz}$ removes all sidebands providing an NMR spectrum with the two peaks at their isotropic chemical shift (C). Simulations were done for a ${ }^{15} \mathrm{~N}$ resonance frequency of $61 \mathrm{MHz}$.

A very elegant and experimentally straightforward way of obtaining narrow NMR spectra of membranes and membrane proteins is magic-angle spinning (MAS) [44]. Here, the sample is contained in a cylindrical rotor and oriented at an angle of $54.74^{\circ}$ with respect to the external magnetic field. By MAS, all spatial spherical tensor components of the CSA Hamiltonian (and of all other second-rank tensors relevant in NMR) are averaged to zero. Thus, all anisotropic broadenings are eliminated and one can obtain the structural information contained in the isotropic chemical shifts [43]. In a nutshell, one can think of MAS as emulating the fast isotropic molecular tumbling of the molecules as it occurs in solution. For a full mathematical description of MAS, the reader is referred to literature $[38,39,45]$. In simple terms, any rotation of the sample about an angle $\theta_{r}$ with respect to the external magnetic field results in a scaling of the anisotropic chemical shift, which can be depicted in the following formula [38]: 


$$
\omega=\omega_{\text {iso }}+\omega_{\text {aniso }} \cdot \frac{1}{2}\left(3 \cos ^{2} \theta_{r}-1\right)
$$

For $\theta_{r}=54.74^{\circ}$, all anisotropic chemical shifts vanish and the NMR frequency detected under MAS conditions equals the isotropic chemical shift. For spinning off the magic angle, residual anisotropic components remain. An example for the effect of MAS on the ${ }^{15} \mathrm{~N} N \mathrm{NM}$ spectrum of a membrane protein with two ${ }^{15} \mathrm{~N}$ labeled amides is shown in Fig. 3 . In the static case, each amide gives rise to a characteristic ${ }^{15} \mathrm{~N}$ NMR powder spectrum but it is already difficult to determine the individual features of each powder pattern in the superposition of the two spectra (A). Introduction of MAS at a slow rotational frequency converts the powder spectrum into a series of narrow peaks, which occur at the isotropic chemical shifts of each site (center bands) and a series of spinning sidebands at integer multiples of the rotational frequency (B). The intensity envelop of these sideband patterns resembles the static powder spectrum. Faster MAS redistributes all sideband intensity into the center band and at a rotational frequency that exceeds the width of the anisotropic chemical shift, only the two isotropic signals are detected (C).

MAS is the by far most used solid-state NMR method for the investigation of membrane proteins and membranes. In principle, many of the successful methods known from solution NMR spectroscopy can be used for assignment, structure determination, and investigation of intermolecular interactions under MAS conditions [46-49]. However, there is one fundamental principle difference. While ${ }^{1} \mathrm{H}$ is almost always detected in solution NMR, the use of the protons in solid-state NMR is limited to cases where the molecules remain highly mobile as relatively small molecules in membranes [22] or by applying MAS frequencies that exceed $60 \mathrm{kHz}$ [50]. The latter is often not practical for the investigation of membranes and membrane proteins as very small MAS rotors are used to achieve high spinning rates that cannot accommodate much sample. Thus, for most MAS applications, nuclei such as ${ }^{13} \mathrm{C},{ }^{15} \mathrm{~N},{ }^{19} \mathrm{~F}$, or ${ }^{31} \mathrm{P}$ are detected, which often necessitates isotopic labeling of the molecules of interest. Further, MAS spectra of these nuclei must be detected under application of high power proton decoupling, as the applied MAS frequencies are typically not sufficient to average out the dipolar couplings of the highly abundant and strongly coupled protons. For MAS investigations of membranes and membrane proteins, MAS rotors with a diameter of 3.2 or $4 \mathrm{~mm}$ are most often used, which provide a sample volume of 20-70 $\mu$ l.

While the dipolar coupling between the nuclei of interest and the abundant protons often represents an unwanted complication of the experiment that is abolished by decoupling, dipolar couplings between nuclear spin pairs are very valuable quantities as they depend on the distance between the interacting nuclei. For an isolated spin pair, the z component of the dipolar coupling tensor, which is typically detected, can be expressed as $[38,39]$ :

$$
D_{\mathrm{zz}}=\frac{\mu_{0}}{4 \pi} \frac{\gamma_{\mathrm{A}} \gamma_{\mathrm{B}} \hbar}{r_{\mathrm{AB}}^{3}}\left(3 \cos ^{2} \Theta-1\right)
$$

where $\mu_{0}$ is the free magnetic inductivity, $\gamma_{A}$ and $\gamma_{B}$ are the gyromagnetic ratios of nuclei $A$ and $B$, respectively, $\hbar$ is Planck's constant divided by $2 \pi$, and $\Theta$ is the angle between the internuclear distance vector and $B_{0}$. Clearly, the dipolar coupling depends on the third power of the nuclear distance vector $r_{A B}$, which can be directly determined from $a$ 
measured dipolar coupling. Thus, dipolar couplings, measured between nuclei on protein and lipid, respectively, are a direct measure of protein-lipid interaction.

Finally, the quadrupolar interaction of the deuterium nucleus has commonly been exploited to study lipid-protein interaction in membranes. ${ }^{2} \mathrm{H}$ is a nucleus with a spin 1 and a relatively small quadrupolar coupling constant. Further, numerous deuterated lipids and amino acids are commercially available and deuterated proteins can also be expressed in $E$. coli relatively easily [51]. Thus, static ${ }^{2} \mathrm{H}$ NMR spectra have widely been acquired to study the influence of membrane proteins on the lipid membrane [52]. The quadrupolar interaction of ${ }^{2} \mathrm{H}$ is caused by the interaction of the quadrupolar moment of the nucleus with the electric field gradient of the chemical bond, which gives rise to a quadrupolar splitting of the NMR signal, which can be described by $[53,54]$ :

$$
\Delta v_{\mathrm{Q}}=\frac{3}{2} \frac{e^{2} q Q}{\hbar} \frac{1}{2}\left(3 \cos ^{2} \Theta-1\right) S_{\mathrm{CD}}
$$

Here, $e^{2} q Q / \hbar$ represents the quadrupolar coupling constant $\left(167 \mathrm{kHz}\right.$ for ${ }^{2} \mathrm{H}$ in the $\mathrm{C}^{2}{ }^{2} \mathrm{H}$ bond) and $\Theta$ is the angle between the membrane normal and the external magnetic field. $S_{C D}$ is the segmental order parameter of the chemical bond. The quadrupolar interaction is strongest of the NMR interactions and for $a^{2} \mathrm{H}$ nucleus in a rigid environment, a quadrupolar splitting of $125.25 \mathrm{kHz}$ is measured. In membrane applications, both the orientation of the chemical bond with respect to the external magnetic field and the molecular dynamics of the bond vector scale the interaction such that only a fraction of the full quadrupolar coupling is detected. From the detected quadrupolar coupling, the orientation and the dynamics of the molecule in the membrane can be determined and has widely been done in the field of membrane biophysics $[42,55,56]$.

With this short summary, the underlying principles of NMR have been sketched. In what follows, we shall introduce the main methods used to study the interaction of membrane lipids with proteins by solid-state NMR. We will distinguish between methods that allow studying the influence of proteins and peptides on the lipid membrane and techniques that directly detect the interaction between proteins and lipids.

\subsection{Solid-state NMR techniques to Study the Influence of Proteins on Lipid}

\section{Membrane Properties}

Internal and membrane-associated proteins do influence the properties of the membrane. This is related to the specific interactions between lipids and proteins $[2,15,57]$, alterations of the lateral pressure profile of the membrane, differences in the hydrophobic thickness between membrane and protein referred to as 'hydrophobic mismatch' [16], the elastic properties of the membrane, or by inducing local curvature in the lipid phase, which has consequences for the phase state of the membrane [15]. Many of these properties can be investigated by solid-state NMR spectroscopy.

\subsection{1 ${ }^{31}$ P NMR Spectroscopy to Study the Influence of Proteins on Membrane Phase} and Structure

${ }^{31} \mathrm{P}$ is an extremely useful nucleus for studying lipid membranes. With a natural abundance of $100 \%$ and just a single occurrence in most phospholipid species, ${ }^{31} \mathrm{P}$ has long been used as a probe for membrane phase state and structure $[40,58,59]$. Static ${ }^{31} \mathrm{P}$ NMR spectra 
showing a powder pattern indicative of an axially symmetric CSA tensor with a span of $\Delta \sigma=\sim 35-55 \mathrm{ppm}$ confirm the presence of a lamellar liquid crystalline membrane phase state for lipid-protein preparations (see Fig. 2D) [60]. Narrow lines at the isotropic NMR frequency, which are superimposed with the powder spectra indicate the presence of highly mobile lipids in the membrane protein preparation and may suggest non-perfect protein reconstitution $[61,62]$. Furthermore, proteins can influence the phase state of the membrane, and ${ }^{31} \mathrm{P}$ NMR spectra are characteristic for lamellar, inverse hexagonal, and isotropic phases [59]. Deconvolution of the ${ }^{31} \mathrm{P}$ NMR spectra can quantitatively determine the relative proportion of these lipid phases of a respective preparation as a function of time $[63,64]$.

${ }^{31} \mathrm{P}$ NMR is also a very reliable technique to probe the degree of orientation and mosaic spread in oriented membrane preparations $[42,65]$. More recently, ${ }^{31} \mathrm{P}$ NMR spectra that show non-perfect orientation of the lipids in the presence of antimicrobial peptides have been interpreted in terms of peptide induced membrane thinning or the formation of toroidal pores [66]. As observed in many studies, oriented ${ }^{31} \mathrm{P}$ NMR spectra in the presence of antimicrobial peptides show low intensity peaks at anisotropic chemical shifts that are indicative of non aligned lipids $[42,67,68]$. Such structures can be induced from phospholipids residing in thinned membranes or pore structures. The effect of membrane thinning can be observed since a portion of the peptide-bound thinned bilayer represents an area of high curvature and, therefore, shows a different orientation and consequently an altered anisotropic chemical shift. Through this method, peptide-induced membrane thinning of $0.5-2.5 \AA$ can be observed [69]. For the analysis, ${ }^{31} \mathrm{P}$ NMR spectra are calculated that also consider the lateral diffusion of lipids in the thinned membranes [66]. Also, lineshape factors that take into account the anisotropic frequency distribution of ${ }^{31} \mathrm{P}$ NMR spectra of lipids that are located in elliptical toroidal pores have been derived $[66,69]$. Thus, with these recent innovations, the relatively 'old' ${ }^{31} \mathrm{P}$ NMR technique has been extended to report on the influence of peptides and proteins on local membrane structure and pore formation.

Finally, ${ }^{31} \mathrm{P}$ MAS NMR spectroscopy has also been used to investigate the influence of membrane binding peptides on the isotropic chemical shifts of the phospholipids through direct protein-lipid contacts $[70,71]$. Peptide induced changes of the individual lipids in mixtures of POPC, POPE, and cardiolipid could be observed as these signals were sufficiently separated. Addition of an N-terminal peptide from the apoptotic Bax protein lead to distinct chemical shift changes of the POPC and cardiolipin lipids but not of POPE [70]. The shifts in the isotropic lipid signals was explained by the ring current effect induced by a Phe residue in the peptide and electrostatic interactions between peptide and the negatively charged cardiolipin [70].

\subsection{2. ${ }^{2} \mathrm{H}$ NMR Spectroscopy to Investigate the Impact of Proteins on Membrane Packing and Lipid Dynamics}

${ }^{2} \mathrm{H}$ is an extremely useful nucleus to study the influence of membrane proteins and peptides on lipid membranes. These days, quite large selections of lipids with specific deuteration in different molecular segments or even almost perdeuterated lipids are commercially available including the major phospholipid species, sphingolipids, gangliosides, or cholesterol. Thus, the impact of membrane proteins on individual lipids in complex mixtures can be investigated by using the deuterated form of the lipid of interest keeping 
the other components of the mixture non-deuterated. The ${ }^{2} \mathrm{H}$ quadrupolar interaction is fairly large to outnumber all other NMR interactions such that clean ${ }^{2} \mathrm{H}$ NMR spectra that show the sole characteristics of the ${ }^{2} \mathrm{H}$ probe in the respective molecular segment are detected, which are straightforward to interpret. While in previous years the influence of membrane proteins on lipid headgroup structure has been studied extensively [37], more recently ${ }^{2} \mathrm{H}$ NMR of chain deuterated lipids makes the largest portion of the studies. Such experiments can be either carried out in multilamellar vesicles with the protein of interest reconstituted in the membranes or in oriented membrane stacks [42]. Chain perdeuterated phospholipids give rise to characteristic ${ }^{2} \mathrm{H}$ NMR spectra that represent the superposition of several Pake doublets, each of which can be assigned to a methylene segment in the lipid chain [53]. From these powder patterns, order parameter plots along the lipid chains are derived and the influence of proteins or peptides on these order parameters is analyzed. As the amplitude of the segmental motions in the lipid chains increases towards the chain end, the order fluctuations increase and consequently smaller order parameters are detected. ${ }^{2} \mathrm{H}$ order parameters can be measured very precisely and already small order variations can be interpreted with regard to alterations in membrane curvature, lipid packing, lipid chain length, and area per molecule $[15,72-75]$. Especially $\alpha$-helical peptides have a profound effect on membrane structure: amphipathic helices insert into the lipid water interface of the membrane and typically increase order of the upper chain segments of the lipids but disorder the lower segments [76]. Transmembrane $\alpha$-helical peptides often induce membrane curvature [15] or membrane hydrophobic mismatch [16] indicated by characteristic changes in the order parameters. Furthermore, ${ }^{2} \mathrm{H}$ order parameters reflect the elastic properties of the membrane and can be used to study the influence of membrane proteins on these properties [73,77-79]. Numerous examples exist in the literature reporting on the influence of membrane proteins and peptides on the membrane lipids studied by ${ }^{2} \mathrm{H}$ NMR [80-88]. But also large membrane proteins influence the properties of the lipids in the membrane as intensely investigated for GPCRs like rhodopsin $[14,15,89,90]$, VDAC [91], or the H+-ATP synthetase subunit c [92].

The same order parameters can also be determined by measurement of the motionally averaged ${ }^{1} \mathrm{H}-{ }^{13} \mathrm{C}$ dipolar couplings using various separated local field experiments under MAS conditions. The molecular vector, which fluctuates and gives rise to averaged ${ }^{2} \mathrm{H}$ quadrupolar or ${ }^{1} \mathrm{H}_{-}^{13} \mathrm{C}$ dipolar couplings is identical and thus identical order parameters are determined. The time scales of both experiments is also similar $(<1 \mu \mathrm{s})$ and thus comparable information can be determined. The determination of dipolar couplings is advantageous when phospholipids with (specific) deuteration are not available. Examples of the recent literature include studies of the membrane disordering effects of an islet amyloid polypeptide [93], $\alpha$-synuclein [94], and MSP containing nanodisks [95].

\subsubsection{Measurement of Lipid and Peptide Diffusion in Membranes}

An other parameter of lipid membranes that can be influenced by lipid-protein interaction is the lateral diffusion of the membrane lipids. As in solution, diffusion in the membrane depends on the size of the diffusing particle and consequently, peptides, proteins, and lipids show clearly different diffusion coefficients. Strong interaction between lipids and proteins or peptides can lead to a decrease in later lipid diffusion, which then can provide a measure for lipid-protein interaction. There are several NMR techniques to measure lateral diffusion in membranes, which have recently been reviewed [96]. A popular method is to 
measure lateral lipid diffusion rates in membranes is the application of pulsed field gradients (PFG) in an echo NMR experiments, which renders the NMR signal diffusion dependent. For diffusion measurements by PFG NMR, the high $\gamma^{1} \mathrm{H}$ nuclei have to be detected, which provides the advantage of spectral resolution allowing to resolve diffusion rates for several molecules in the membrane $[30,75]$. However, larger molecules are characterized by strong ${ }^{1} \mathrm{H}-{ }^{1} \mathrm{H}$ dipolar interactions, which deteriorates spectral resolution. Two approaches have been developed to circumvent this problem, on is the combination of PFG NMR with MAS [97] or the macroscopic alignment of the lipids between glass plates [98]. Alternatively, two-dimensional exchange spectroscopy (EXSY) using ${ }^{31} \mathrm{P}$ NMR has been employed to study lateral lipid diffusion either in static samples [99] or by using the CODEX MAS NMR technique [100]. The latter methods do not require the application of pulsed field gradients and can be implemented on standard NMR spectrometers with a standard MAS probe. The CODEX NMR technique is also more sensitive than the static EXSY technique that detects powder NMR line shapes.

Although a quite useful technique, there have not been very many papers on the influence of membrane proteins or peptides on the lateral diffusion of the membrane lipids $[101,102]$. Orädd et al. have studied the influence of the artificial antimicrobial peptide CNY21 on lipid diffusion in POPC membranes and found that the peptide that penetrates the lipid membrane slows down lipid diffusion [103]. The reduction of the lipid diffusion coefficients in the presence of the peptide was attenuated in the presence of cholesterol.

\subsubsection{The Influence of Membrane Proteins on Lipid Dynamics Measured by Relaxation} Briefly, we will mention that membrane proteins and peptides also influence the dynamic properties of the lipids and this effect can be monitored by measuring nuclear relaxation rates. As mentioned in the beginning, the lipids in a membrane are highly dynamic molecules that undergo large amplitude motions on several time scales $[42,47]$. While the amplitudes of these motions are easily determined by the ${ }^{2} \mathrm{H}$ or ${ }^{1} \mathrm{H}^{-13} \mathrm{C}$ order parameters (vide supra), the measurement and analysis of spin relaxation rates represents a less used technique. Excited spin states relax back to equilibrium in a stochastic process that can be characterized by a specific time constant called relaxation time. Thus, molecular reorientations, fluctuations, internal motions, or chemical exchange influence relaxation rates and the analysis of the latter provide a detailed picture of the correlation times and amplitudes of these specific motions. Most useful for studying the influence of proteins on lipid motions are the relaxation rates of ${ }^{2} \mathrm{H}$ and ${ }^{13} \mathrm{C}$. Especially for ${ }^{2} \mathrm{H}$ relaxation of Zeeman $\left(R_{1 z}\right)$ and quadrupolar order $\left(R_{10}\right)$, the theory has been worked out in particular for oriented membrane preparations $[28,29,104]$. For ${ }^{13} \mathrm{C}$ relaxation typically detected under MAS conditions, modified Lipari-Szabo approaches are useful $[36,105]$. Nevertheless, only few reports are found in the literature, for instance on the influence on rhodopsin on lipid relaxation rates [106]. What is observed very often, but rarely analyzed quantitatively is the fact the in the presence of membrane proteins, the NMR signals of the lipids become broader due to an increase in the correlation time of lipid motion, which decreases $T_{2}$ relaxation, which is observed in an increase in line width $[60,85,95,107]$.

2.3. Solid-State NMR Methods for the Direct Investigation of Protein - Lipid Interaction Traditionally, the power of NMR lies in the ability to directly observe the interaction between nuclei in both intra- and intermolecular structures. This general virtue of NMR is also exploited in the investigation of protein-lipid interactions. Starting with the nuclear 
Overhauser effect (NOE) between protons and dipolar recoupling of interacting spins of lipids and proteins, new methods based on spin diffusion, the interaction of nuclear spins with electron spins, or the transfer of magnetization have been developed in the last 15 years that allow to investigate the interaction between proteins an lipids rather directly and quantitatively. All these methods utilize MAS, which allows resolving the NMR signals of membrane lipids as well as those of the protein.

\subsubsection{Nuclear Overhauser Enhancement Spectroscopy (NOESY) of Peptide-Lipid Dispersions}

The basis for most NMR structure determination is the nuclear Overhauser effect (NOE), which allows the determination of ${ }^{1} \mathrm{H}-{ }^{1} \mathrm{H}$ distances of up to $5 \AA$ [108]. An NOE can also be observed between membrane bound peptides and lipids [17,109]. However, a few alterations to the use of the NOE in the determination of the structure of proteins or nucleic acids in solution apply [110]. First, the most important point to remember is that the NOESY experiment in lipid-peptide studies detects intermolecular contacts. Even within lipid membranes, NOESY derived cross-relaxation rates represent mostly contributions from intermolecular contacts [22], which can occur between molecular segments that might be separated by $20 \AA$ or more $[23,25]$. Due to the high molecular dynamics in lipid membranes, NOESY contacts cannot be interpreted as fixed distances but rather represent a distribution of distances that are modulated by various correlation times that represent the multitude of molecular motions in lipid membranes [25]. Due to the $r^{-6}$ dependence of the NOESY cross-relaxation rates, the shortest distance between interacting spins will contribute the largest portion to the effect. Thus, NOESY cross-relaxation rates can be considered as contact probabilities between the segments of the interacting molecules and should never be interpreted as fixed distances between lipids and peptides [22].

Second, NOESY interaction studies between peptides and membrane lipids require application of fast MAS $\left(\omega_{r} / 2 \pi>10 \mathrm{kHz}\right)$ and only work well for relatively mobile small peptides of no more than 10 to 15 residues $[17,111]$. The reason for this limitation arises from the fact that the very dense ${ }^{1} \mathrm{H}$ network in the peptide of interest represents a complicated homogeneous dipolar interaction that can only be averaged out by MAS with a frequency that exceeds the interaction strength, which is on the order of $50 \mathrm{kHz}$. While such MAS rates can be achieved in small MAS rotors, these containers only contain a very small volume and often are not practical for the investigation of aqueous lipid-peptide dispersions. However, peptides that remain sufficiently mobile in the membrane or at the membrane surface often undergo axially symmetric reorientations and, therefore, the dipolar broadening is reduced to an inhomogeneous broadening, which can be averaged out at lower MAS frequencies [17] and thus lipid-peptide interactions can be observed.

A third complication arises from the fact that the lipid-protein ratio in the sample favors the detection of the lipid rather than the peptide signals, the latter being broadened due to efficient relaxation processes in the less mobile membrane peptides. While deuteration of the phospholipids partially solves this problem, the NOESY NMR method is more suited to study the localization of small molecules in the membrane [112-115] and has only been used in selected cases for peptide-lipid interactions. Some studies have also used ${ }^{1} \mathrm{H}-{ }^{1} \mathrm{H}$ recoupling techniques during the mixing time to improve the intensity of the lipid-peptide cross peaks [116-118]. 
For example, the localization of a lipid-modified heptapeptide representing the membrane anchor of the human N-Ras protein has been investigated in almost perdeuterated DMPC$d_{67}$ membranes $[119,120]$. In these experiments, backbone and side chain signals of the peptide could be assigned in the ${ }^{1} \mathrm{H}$ MAS NMR spectra and NOEs between the undeuterated glycerol and upper chain segments of the phospholipids were observed. Much weaker NOEs to the lipid chain ends indicated a localization of the very mobile Ras peptide in the lipid-water interface of the membrane.

\subsubsection{Spin Diffusion from Lipids into Membrane Proteins}

From the last section, it is obvious that the detection of the abundant ${ }^{1} \mathrm{H}$ nuclei of membrane proteins is much less straightforward than in soluble proteins. Therefore, in solid-state NMR studies of membrane proteins and for the detection of lipid-protein contacts, most often the heteronuclei $\left({ }^{13} \mathrm{C},{ }^{15} \mathrm{~N}\right.$, or $\left.{ }^{31} \mathrm{P}\right)$ are detected unless the protein of interest is highly deuterated [51]. One way of studying lipid-protein interaction is the detection of magnetization on the protein that originates from the lipids of the membrane and diffuses into the protein by spin diffusion. Thus, techniques that detect lipid originated magnetization on the protein directly represent a simple yet useful tool to observe lipidprotein interaction.

This technique was first demonstrated in 2002 using a pulse sequence that creates selective magnetization on the phospholipid molecule that is transferred into the protein by ${ }^{1} \mathrm{H}$ spin diffusion [121]. Magnetization is transferred to ${ }^{13} \mathrm{C},{ }^{15} \mathrm{~N}$, or ${ }^{31} \mathrm{P}$ in a short $\mathrm{CP}$ or Lee Goldburg CP step [34]. Only protein magnetization is detected that was transferred from the lipid, which, thus, represents a true measure of protein-lipid interaction. The experiment can be carried out in a two-dimensional fashion, such that the ${ }^{1} \mathrm{H}$ dimension is incremented before the spin diffusion period. Thus, the source of spin diffusion (i.e., the molecular segment of the individual lipid species, from which spin diffusion is originated) as well as the sink (i.e., the protein segment that receives the magnetization) the can be determined. This experiment was first demonstrated on the channel domain of the membrane toxin colicin la [121]. As this membrane protein contains a transmembrane segment, spin diffusion build up into the protein is fast as oppose to a protein that is only surface bound. In the latter case, much slower spin diffusion build up is observed as magnetization has to travel within the lipid, which is a slow process due to the molecular dynamics that weakens the dipolar couplings [122].

Spin diffusion from lipid into membrane proteins or peptides has been excessively used mostly to detect the topology of antimicrobial and fusion peptides [64,123-126] and segments of larger membrane binding proteins such as GCAP-2 [127]. Further, interactions between POPC in the lipid-protein boundary to the membrane scaffolding protein in nanodisks have been observed in a 3D version of the spin diffusion experiment [128].

The direct interaction between lipids and the S1-S4 voltage sensing domain of an archaebacterial voltage-activated potassium channel have been investigated [129]. In ${ }^{15} \mathrm{~N}$ detected spin diffusion experiments, the interaction of the amides as well as the side chains of Arg residues of the voltage sensing domain with the membrane lipids (POPC and POPG) could be shown. Lys residues showed much weaker interaction with the membrane interior. However, due to strong signal superposition between the PC and PG in the membrane, the interaction between the protein and either of the lipid species could not 
further be specified. However, when the S1-S4 domain of Kv was reconstituted into DOTAP, a positively charged model lipid that lacks the phosphodiester group, somewhat slower spin diffusion build up from the lipid into the amides and in particular into the $\operatorname{Arg} \mathrm{N}^{\eta}$ side chain was observed [129]. Nevertheless, the structural integrity of the protein in DOTAP membranes was confirmed.

\subsubsection{The Investigation of Lipid-Protein Interactions by Saturation Transfer NMR}

The well-established concept of measurement of saturation transfer from soluble proteins to a ligand in solution [130] can also be exploited for the study of lipid-protein interactions in membranes [14]. In saturation transfer experiments in membranes, magnetization from the lipid is transferred to the membrane protein. Membrane protein resonances are selectively saturated and the transfer of magnetization due to spin diffusion to the bound lipids is observed. The lipid-protein contacts are then detected through intensity changes of the lipid resonances and typically a saturation transfer difference (STD) spectrum is analyzed. Lipid segments or lipid species that are strongly interacting with the protein are identified in the STD spectrum while non-interacting molecules show no intensity. From these spectra, rates of magnetization transfer can be determined that quantify the interaction strengths. Transferred magnetization on the interacting lipids can be detected via ${ }^{1} \mathrm{H}$, but can also be transferred to ${ }^{13} \mathrm{C}$ for better resolution or to ${ }^{31} \mathrm{P}$ to distinguish between lipid species provided they can be resolved in a 1D NMR spectrum.

Very nice applications of this technique have been demonstrated for phospholipidrhodopsin interactions $([131,132]$, vide infra) and for the interaction of S1-S4 domain of Kv with lipids [129].

2.3.4. The Investigation of Lipid-Protein Interactions by Dipolar Coupling Measurement As clear from eq. (3), there is a simple relationship between the dipolar coupling between neighboring nuclei and their distance; thus, dipolar coupling measurement in solid-state NMR represents a direct way of studying lipid-protein interactions. A technical difficulty arises from the fact that the detection of highly resolved spectra of lipids and proteins in the membrane necessitates application of MAS, which averages out the dipolar couplings of interest. This complication has been overcome by the recoupling of the spin interaction of interest allowing to observe selected dipolar couplings or other anisotropic parameters that are averaged by MAS $[133,134]$. Such measurements are best carried out as twodimensional separated local field experiments that allow detecting the highly resolved MAS spectrum in one dimension and the NMR spectrum that features the classical line shapes of the anisotropic interactions in the other [135]. The prototypes of experiments for homoand heteronuclear dipolar coupling measurements are rotational resoncance [136] and REDOR [137], respectively. In such experiments, ${ }^{13} \mathrm{C}-{ }^{15} \mathrm{~N}$ and ${ }^{13} \mathrm{C}-{ }^{13} \mathrm{C}$ distances of up to $\sim 4.5 \AA$ and $\sim 6 \AA$ can be detected, respectively. For the interaction of lipids and proteins, determination of ${ }^{13} \mathrm{C}-{ }^{31} \mathrm{P}$ contacts can be useful, which are measurable up to $7 \AA$ due to the higher gyromagnetic ratio of ${ }^{31} \mathrm{P}$. In the last decade, M. Hong and $\mathrm{K}$. Schmidt-Rohr also developed new NMR techniques that allow measuring ${ }^{1} \mathrm{H}-\mathrm{X}$ heteronuclear dipolar couplings of up to $8 \AA$ while suppressing unwanted ${ }^{1} \mathrm{H}^{-1} \mathrm{H}$ couplings [138]. Provided a ${ }^{19} \mathrm{~F}$ nucleus can be incorporated into the lipid or protein of interest without disturbing the membrane or protein structure, the distance that can be measured reliably can even exceed $10 \AA$. In addition to these dipolar coupling measurements, methods that use selective 
depolarization of protein magnetization by lipids have been developed for the observation of lipid-protein interactions [139].

There are numerous examples in the literature that document the usefulness of direct dipolar coupling measurements for lipid-protein or lipid-peptide interactions in membranes. Recent examples include the study of peptide-lipid interactions of the antimicrobial peptides PG-1 [140,141], K3 [142], or lactoferrampin [143], a fusion peptide from HIV [144], the N-terminal region of the pore-forming toxin equinatoxin II [145], or cellpenetrating peptides [68]. In particular, the formation of salt bridges between the lipid's phosphate and the side chain of positively charged amino acids could be demonstrated [146].

While direct distance measurements via dipolar couplings ideally require isolated spin pairs, typically involving selective isotopic labeling, solid-state NMR experiments may also detect complex correlation maps of all interaction spins in a membrane protein similar to the NOESY experiment in solution. Such experiments can be carried out in either homonuclear or heteronuclear fashion and provide a fingerprint of the complex inter- and intramolecular dipolar interactions. Thus, also lipid-protein interactions can be probed in the membrane. The standard ${ }^{13} \mathrm{C}-{ }^{13} \mathrm{C}$ experiment utilizes proton-driven spin diffusion for magnetization exchange [147], but numerous recent developments have introduced various different mixing schemes utilizing recoupling [148]. While lipid molecules are often non-isotopically labeled, highly abundant, and in fast exchange between a free and a protein bound state, the detection of lipid-protein contacts in membrane proteins is challenging. However, nonannular lipids, which bind to membrane proteins with high affinity and are also detected in crystal structures have been detected using ${ }^{13} \mathrm{C}-{ }^{13} \mathrm{C}$ correlation NMR experiments ([149] vide infra).

\subsubsection{The Interaction of Lipid-Attached Spin Probes with Membrane Proteins}

Paramagnetic enhancement of nuclear relaxation times in proteins is a method that has widely been used in structure determination of soluble proteins [150]. Also in solid-state NMR of membrane proteins, paramagnetic labels covalently attached to lipid molecules can be used to study lipid-protein interactions. The unpaired electron on the spin label induces fast relaxation of a nuclear spin on the protein, which gives rise to a paramagnetic relaxation rate that can be determined from a simple relaxation time measurement. This rate shows a $r^{-6}$ dependence of the distance between electron and nuclear spins. As this intermolecular distance is fluctuating as a result of the high dynamics in lipid membranes, caution in the interpretation of distances is advised and the parameter is better interpreted as a general contact probability [151]. It should also be kept in mind that similar to lipid attached fluorescence labels spin probes also show a rather broad distribution functions in the membrane [152-154], which renders interpretation of paramagnetic relaxation rates difficult. Nevertheless, with the available spin labeled phospholipid [155] or the cholesterol analogs a number of lipid species are available for studying lipid-protein interaction in membranes [153].

While the technique of paramagnetic relaxation enhancement has been used to study the interaction of the voltage sensor domain from KvAP with phospholipids in phospholipid containing detergent micelles [156], application of this technique in lipid bilayers tend to 
focus on smaller peptides. For instance, the membrane interaction of neuropeptide $Y$ $[157,158]$ or calcitonin [159] was studied.

\section{What has been Learned?}

After the general introduction of the methods used in solid-state NMR to study lipid-protein interaction in membranes, we will continue to discuss a few seminal examples, where these techniques have been used. We will confine the discussion to four examples from the recent literature that show individual aspects of lipid-protein interaction in membranes.

\subsection{The Interaction of the Ras Lipid Modifications with Lipid Membranes of \\ Varying Thickness}

Membrane interaction is crucial for the human Ras proteins, which represent a molecular switch in several important signal transduction cascades in biology [160]. The lipid modified C-terminal membrane binding domain of the $\mathrm{N}$-Ras protein forms a dynamic horseshoe like structure [36,161] and is localized in the lipid-water interface of the membrane [119]. Membrane binding of the protein is facilitated by insertion of a farnesyl moiety at Cys 186 and a palmitoyl chain at Cys 181 into the membrane along with the membrane interaction of several hydrophobic resides [160]. This raises the question how deep these lipid modifications can reach into the membrane and how the membrane structure can influence the insertion process. The adaptation of the Ras lipid chains was studied in membranes of varying chain composition, including DLPC with a hydrophobic thickness of 21.0 ̊, DMPC (25.8 ̊), POPC (28.6 $)$ ), and DPPC/cholesterol (38.8 $)$ [162]. Lipid chain order of the host membrane in the absence and in the presence of Ras as well as of Ras with a deuterated palmitoyl chain were determined using ${ }^{2} \mathrm{H} N M R$. While the lipid chain order of the membrane did not noticeably change in the presence of Ras, the ${ }^{2} \mathrm{H} N M R$ spectra of Ras in the membrane underwent rather drastic changes in response to the various membrane environments. Relatively narrow ${ }^{2} \mathrm{H}$ NMR spectra of the Ras chains were detected in DLPC, indicative of a highly disordered peptide lipid chain (Fig. 4A-C). In contrast, the Ras lipid modifications in the rigid DPPC/cholesterol membrane showed very broad ${ }^{2} \mathrm{H}$ NMR spectra indicating a highly ordered lipid chain. Interestingly, the conversion of the ${ }^{2} \mathrm{H}$ order parameters into chain lengths revealed that Ras adopted the length of its palmitoyl chain exactly to that of the surrounding host membrane irrespective of its hydrophobic thickness (see Fig. 4D). This showed that the Ras protein can vary the length of the palmitoyl chain between 8.7 and $15.5 \AA$ to match the hydrophobic thickness of the surrounding membrane. 

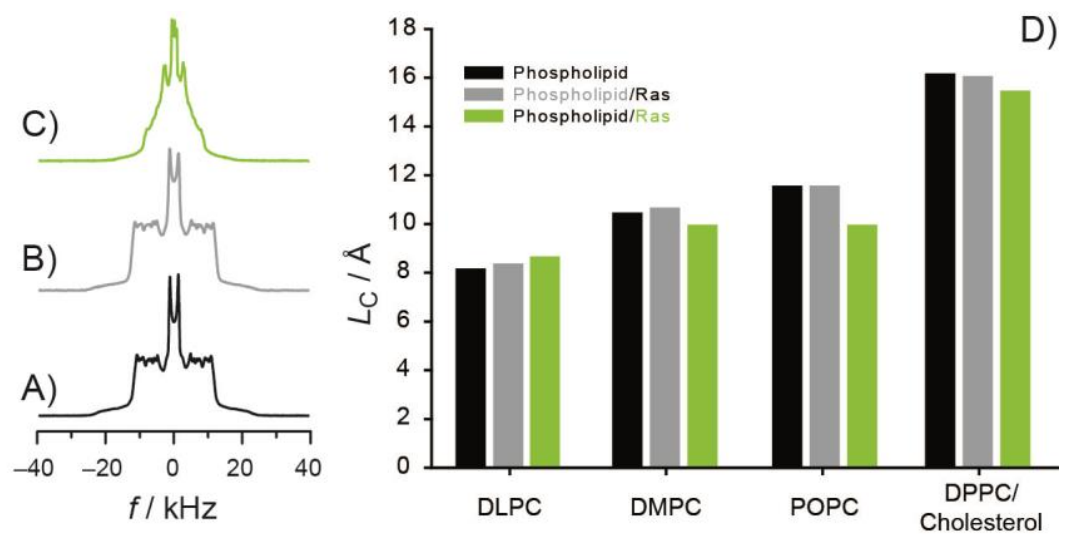

Figure 4. ${ }^{2} \mathrm{H}$ NMR spectra of deuterated DLPC (A), deuterated DLPC- $\mathrm{d}_{46} / \operatorname{Ras}(10: 1)(B)$, and deuterated Ras- $d_{66}$ in DLPC (C). Chain lengths of the phospholipids (black and gray) and Ras peptides (green) in host membranes of varying composition $\left(30^{\circ} \mathrm{C}\right)$.

What appears to be an interesting physicochemical chain matching phenomenon is also of biological importance. In the cell, Ras travels between the plasma and Golgi membranes and within different compartments of the plasma membrane with varying hydrophobic thicknesses. The lipid anchor on the $\mathrm{N}$-Ras protein controls the fast and reversible distribution of the molecule over the various membranes [163]. Protein palmitoylation is also a raft targeting signal, and Ras is targeted to rafts as well [164]. The raft (liquidordered, $\left.I_{0}\right)$ and liquid-disordered $\left(I_{d}\right)$ domains of the membrane are characterized by different thicknesses $[21,24] .{ }^{2} \mathrm{H}$ NMR, AFM, and fluorescence microscopy studies showed that Ras was localized in the $I_{d}$ domains of raft forming biological membranes and that a sizable portion of the protein was directly found at the interface between $I_{\mathrm{o}}$ and $I_{\mathrm{d}}$ domains. Thus, ${ }^{2} \mathrm{H}$ NMR could identify the important influence of the membrane to the structure and dynamics of the Ras lipid chains that represent a prerequisite for the sorting and trafficking of the protein in cellular membranes.

\subsection{Membrane Interactions of the Cationic Antimicrobial Peptide Protegrin-1}

As one out of many examples in the literature for studies of the interaction of membraneactive peptides with lipids, we will discuss recent work on the peptide protegrin-1 (PG-1). More detailed reviews on solid-state NMR studies of antimicrobial peptides have been published recently [165-167]. PG-1 is a cationic antimicrobial peptide found in porcine leukocytes that permeabilizes the lipid membrane of microbes and causes cell death [168]. The peptide structure and dynamics in the membrane has been intensely studied by solidstate NMR in the group of M. Hong $[122,123,165,169]$. PG-1 assumes a $\beta$-hairpin structure, which is stabilized by two intramolecular disulfide bridges. Furthermore, direct investigations of lipid-protein interactions have been reported. In the course of action, the antimicrobial peptide has to translocate across the cell membrane, which is energetically highly unfavored as the insertion of the positively charged sidechains into the membrane is energetically very costly $[170,171]$. 
A)

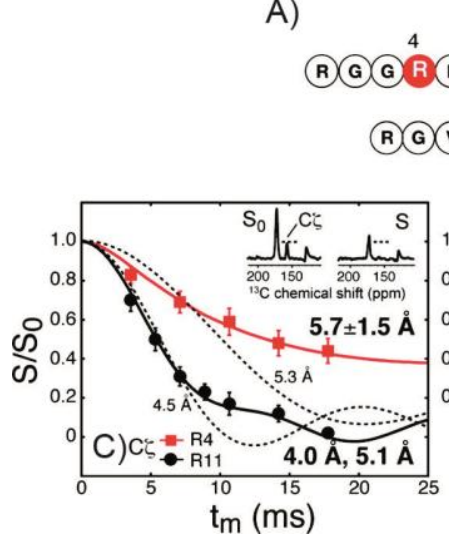

B)
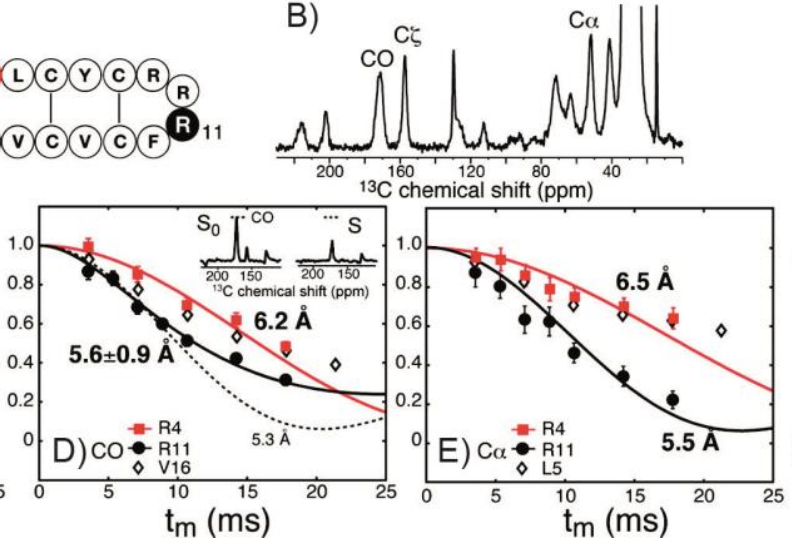

F)

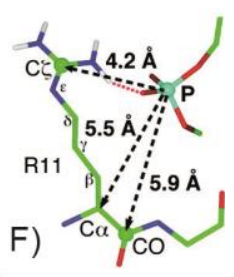

Figure 5. Summary of ${ }^{13} \mathrm{C}-{ }^{31} \mathrm{P}$ REDOR distance measurements between $\mathrm{PG}-1$ and membrane lipids. (A) Schematic sequence of PG-1, (B) exemplary ${ }^{13} \mathrm{C}$ CPMAS NMR spectrum of $\mathrm{Arg}_{4}$ labeled PG-1 in POPE/POPG membranes, (C-E) ${ }^{13} C_{-}{ }^{31}$ P REDOR dephasing curves of several labeled amino acids in PG-1 showing the dephasing of $C \zeta$ signal (C), $C O$ signal (D), and $C \alpha$ signal (E) with the respective distances to the phosphate group as determined from the fit. (F) Model of the guanidinium-phosphate complex for $\operatorname{Arg}_{11}$ showing the measured ${ }^{13} \mathrm{C}^{-31} \mathrm{P}$ distances. A putative hydrogen bond between the guanidinium and phosphate groups is indicated as a red dotted line. Reprinted with permission from [169]. Copyright (2007) American Chemical Society.

To understand the mechanism of this translocation, Hong and coworkers measured ${ }^{13} \mathrm{C}-{ }^{31} \mathrm{P}$ distances between the Arg-rich PG-1 and the phospholipids using ${ }^{13} \mathrm{C}_{-}{ }^{31} \mathrm{P}$ REDOR techniques. Surprisingly, for all investigated residues, including two Arg scattered throughout the peptide sequence, short distances between the peptide and the phosphate groups of the phospholipids were found [169] (Fig. 5). Typical distances of peptide side chains as well as the backbone to the lipids were 4.0 to $6.5 \AA$ in anionic POPE/POPG membranes and 6.5 to $8.0 \AA$ in zwitterionic POPC bilayers. The shortest distances were found between the guanidinium $C \zeta$ nucleus of $\operatorname{Arg}_{11}$ in the $\beta$-turn of PG-1 and the phosphate group suggesting the formation of a hydrogen bond. The PG-1 topology was further studied by ${ }^{1} \mathrm{H}$ spin diffusion rates from the phospholipid chains into the headgroup in the presence and in the absence of PG-1. As PG-1 bridges the headgroup chain distance in the lipid, faster spin diffusion build up was observed. As a result of lipid binding, it was found that PG-1 slows down the lateral diffusion of lipids [172]. In agreement with the specific interactions of the Arg residues of PG-1 with the lipid's phosphate groups are results on a charge-attenuated PG-1 mutant (termed $\left[\Delta_{4,18} G_{10}\right]$ PG-1) that shows longer distances to the lipid headgroups as the WT as determined by REDOR measurements [141]. Also, the depth of insertion of this mutant strongly depended on the membrane surface charge density and in a zwitterionic POPC membrane, the PG-1 mutant remained on the membrane surface as inferred from very different spin diffusion build up curves in POPC and POPG membranes, respectively [141].

The authors concluded that the cost of inserting the positively charged group into the hydrophobic membrane is compensated for by the neutralization of the guanidinium ion by the surrounding negatively charged phosphate groups $[140,169]$. The data indicated that membrane-embedded PG-1 causes some phosphate groups to insert into the membrane interior to come in close proximity to the Arg residues. Such a scenario would also be in agreement with the formation of a toroidal pore, where rotated lipids are in direct contact 
with the entire sequence of the peptide. ${ }^{31} \mathrm{P}$ NMR line shapes confirm the presence of such pores $[173,174]$.

A similar methodology was also used to study the topological structure of PG-1 in a lipopolysaccharide (LPS)-rich membrane mimicking the outer membrane of Gram-negative bacteria [64]. While the spin diffusion data indicated that PG-1 was fully inserted in to the LPS-rich membrane, the ${ }^{13} \mathrm{C}-{ }^{31} \mathrm{P}$ distances between the most inserted $\operatorname{Arg}_{11}$ and the phosphates were larger than in POPE/POPG membranes, indicating that PG-1 forms barrelstave pores in LPS-rich membranes rather than toroidal pores [64].

\subsection{The Specificity of Phospholipid Interaction of the G Protein-Coupled Receptor Rhodopsin}

G protein-coupled receptors (GPCRs) represent the largest class of membrane proteins and mediate numerous cellular processes. Although in the last 5 years crystal structures of several GPCRs have been solved, the by far best studied GPCR is rhodopsin, the biological pigment of photoreceptor cells. For rhodopsin, crystal structures in the ground and in the activated state are available [175-178]. The influence of the membrane properties on the function of rhodopsin has been intensely investigated by various NMR methods in the laboratories of K. Gawrisch and M. F. Brown $[14,15]$. Two aspects have been in the focus of these investigations: the influence of rhodopsin on the properties of the surrounding membrane and specific lipid-rhodopsin interactions.

A first example for the usefulness of precise membrane thickness measurements by ${ }^{2} \mathrm{H}$ NMR involves a study of the $\alpha$-helix content of rhodopsin reconstituted into a series of mixed-chain lipids with varying chain lengths from 14 to 20 carbons and a saturated $s n-1$ and a monosaturated sn-2 chain [107]. It was shown that the rhodopsin structure and function are very sensitive to alterations in membrane hydrophobic thickness and that the $\alpha$-helical content of rhodopsin increases with bilayer thickness suggesting a high plasticity of the rhodopsin transmembrane helices for matching the membrane hydrophobic thickness [107].

Upon activation, rhodopsin induces elastic deformations of the membrane due to hydrophobic mismatch between the thickness of the membrane and the protein, which induces membrane curvature, and the adaptation of lipid molecules to shape changes of the protein upon activation $[14,15]$. It has been found that the nonlamellar-forming lipid phosphatidylethanolamine (PE) supports the formation of the metarhodopsin II (MII) photointermediate [179]. It was also suggested that MII has a larger hydrophobic thickness than metarhodopsin I (MI) [179]. The elastic remodeling of the membrane upon rhodopsin acitvation has been investigated intensely $[89,179,180]$.

${ }^{2} \mathrm{H}$ NMR spectroscopy has further contributed to understand the influence of the lipid matrix on the MI/MII equilibrium of rhodopsin. From the ${ }^{2} \mathrm{H}$ NMR order parameters, the precise bilayer hydrophobic thickness could be measured from which changes in the membrane bending rigidity could be determined. Thus, the observed shifts in the MI-MII equilibrium of rhodopsin in membranes containing a series of PEs with different levels of headgroup methylation and varying chain unsaturation could be correlated with the membrane elastic properties. Interestingly, the shifts in the MI-MII equilibrium could only partially be explained by elastic energy and contributions from hydrogen bonding between 
PE headgroups and rhodopsin had to be invoked to explain the data [180]. In a nutshell, the negative curvature elastic stress exerts the dominating influence on the MI/MII equilibrium $[89,179]$. The influence of direct interactions with the first lipid layer is of secondary importance.

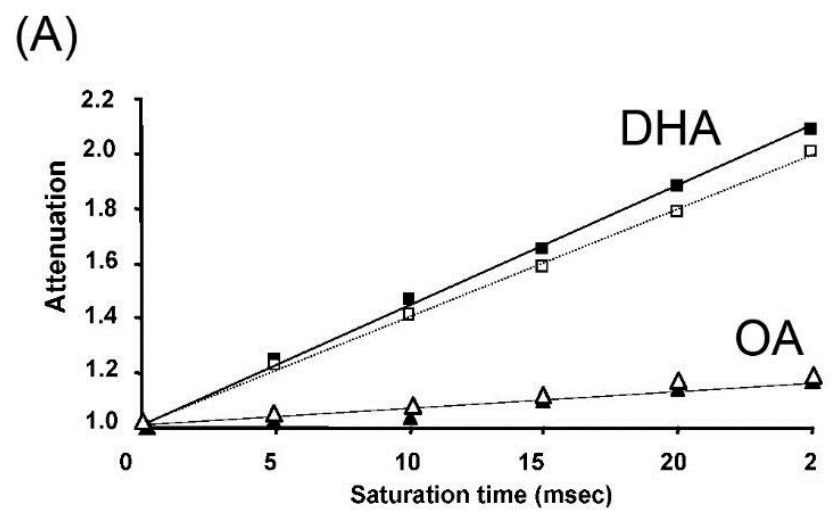

(B)

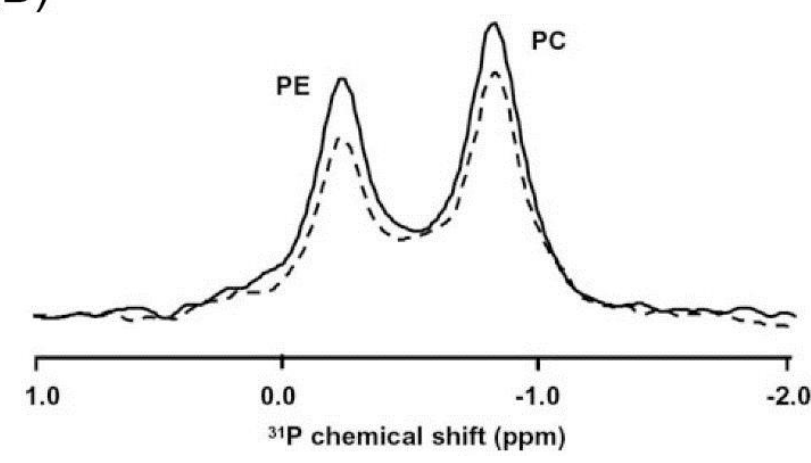

Figure 6. Summary of STD experiments of reconstituted bovine rhodopsin in membranes. (A) Saturation time dependence of the attenuation factor of oleic acid (OA, triangles) and docosaexaenoic acids (DHA, squares) in POPC/rhodopsin (250:1) and SDPC/rhodopsin (250:1) (filled symbols) as well as in POPC/SDPC/rhodopsin (125/125/1) (open symbols). In all cases, the DHA chains show stronger signal attenuation due to preferential interaction with rhodopsin. In all lipid mixtures, the saturated sn-1 chain of the phospholipids was perdeuterated. Reproduced from [131] with permission from the author. (B) 31P MAS STD NMR spectra of bovine rod outer segment disks. Spectra were obtained without (solid line) and with (dashed line presaturation of rhodopsin. Both PC and PE are presaturated but the NMR signal of PE is more attenuated than that of PC, indicating preferential interactions of rhodopsin with PE lipids. Reproduced from [132] with permission from the author.

These results triggered the Gawrisch group to investigate the direct interaction between rhodopsin and phospholipid molecules. Saturation transfer difference was used to determine the phospholipid hydrocarbon chain [131] and headgroup selectivity of rhodopsin in artificial membrane mixtures and native disk membranes [132]. The STD curves suggested that rhodopsin had a preference for polyunsaturated docosahexaenoic acid (DHA) chains over saturated or monounsaturared chains of phosphatidylcholine (PC) membranes suggesting a radial distribution of lipids with the polyunsaturated chains oriented preferentially towards the transmembrane helices of rhodopsin [131] (Fig. 6A). STD experiments with ${ }^{31} \mathrm{P}$ detection were carried out in rod outer segment (ROS) disk membranes to investigate a possible headgroup preference of bovine rhodopsin. The detected ${ }^{31}$ P MAS NMR spectra allowed resolving the PE and PC component of the ROS. The signal intensities of both lipid species were attenuated after rhodopsin presaturation, 
however, the PE signal lost more intensity suggesting a preference of the GPCR for this lipid [132] (Fig. 6B). This data is in agreement with a heterogeneous lipid-rhodopsin interface in the membrane. All photointermediates of rhodopsin transferred magnetization preferentially to the DHA-containing lipids, suggesting a unique preference of the protein for polyunsaturated phospholipid chains.

\subsection{The Interaction of the $K^{+}$Channel KCSA with Tightly Bound Phospholipids}

The procaryotic homotetrameric potassium crystallographically-sited channel (KcsA) is a potassium ion channel from Streptomyces lividans that is activated by alterations in the ambient $\mathrm{pH}$ [181]. Crystallographic work has identified electron density indicative of a tightly bound lipid molecule, identified as a negatively charged phosphatidylglycerol (PG), which was found in each of the four segments of the tetramer [182]. It was shown that lipids (not necessarily PG) were required for the correct refolding of the channel into its functional form. However, ion conduction could only be observed when the protein was reconstituted into vesicles containing negatively charged lipids.
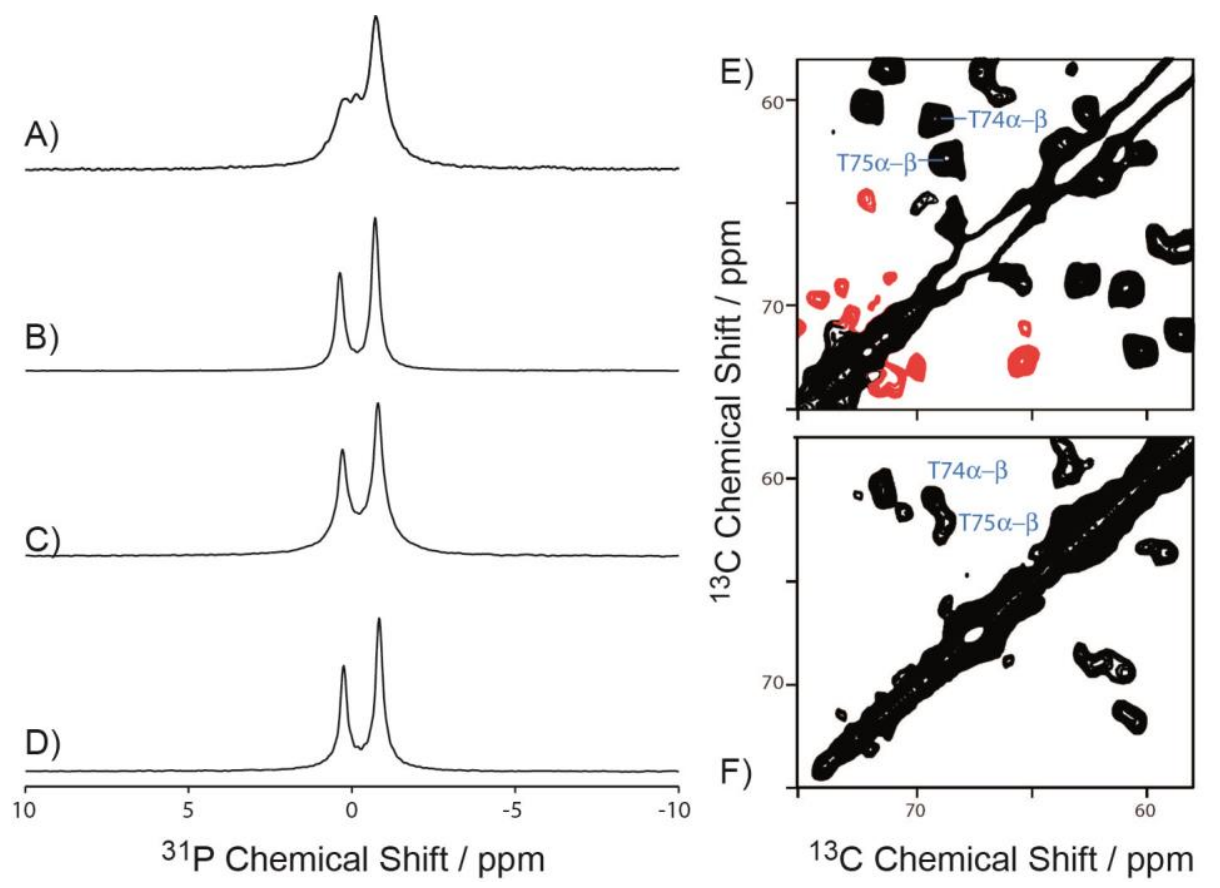

Figure 7. NMR investigations of phospholipids tightly bound to the $\mathrm{K}^{+}$channel $\mathrm{Kcs} A$. (A) protondecoupled ${ }^{31}$ P NMR spectra of WT KcsA. The site directed mutations R64L (B), R89L (C), and R64L/R89L (D) are shown, all spectra show the $\mathrm{K}^{+}$channel reconstituted into POPC/POPG membranes at a mixing ration of $70 / 30(\mathrm{~mol} / \mathrm{mol})$. (E) Sections of a $2 \mathrm{D}{ }^{13} \mathrm{C}-{ }^{13} \mathrm{C}$ correlation NMR spectra of KcsA (E) and LVWY reverse-labeled KcsA Kv1.3 (F) to probe for tightly bound lipids. Lipid headgroup crosspeaks, copurified from E. coli, are highlighted in red. Reproduced from [183] with permission. Reprinted with permission from [149]. Copyright (2007) American Chemical Society.

The direct detection of the interaction between non-annular binding sites on the protein and anionic lipids has been demonstrated using ${ }^{31} \mathrm{P}$ MAS NMR spectroscopy [183]. The channel tetramer was reconstituted into zwitterionic POPC and negatively charged POPC/POPG (70/30) membranes. Significant changes in the ${ }^{31} \mathrm{P}$ MAS NMR spectra were observed in the presence of the ion channel. In the presence of KcsA, the line width of the 
POPC signal in pure POPC membranes increased. In the mixed POPC/POPG membrane, a third ${ }^{31} \mathrm{P}$ NMR signal was detected in addition to the signals for the bulk POPC and POPG, respectively (Fig. 7A). This new component accounted for $6.6 \%$ of the total intensity and was accompanied with a slight reduction of the POPG intensity. This peak was assigned to bound POPG at the non-annular binding site of KcsA, which was expected to account for $4 \%$ of the total lipid intensity at the reconstitution ratio of 100:1 lipid/protein monomer. To confirm that the newly found peak in the lipid spectrum indeed represented a bound lipid, a series of mutants of the KcsA channel were studied. In the crystal structure, it had been proposed that that residues $\operatorname{Arg}_{64}$ and $\operatorname{Arg}_{89}$ were involved in non-annular lipid binding [182]. These residues were mutated to leucine in two single and one double mutant [183]. In the ${ }^{31} \mathrm{P}$ MAS NMR spectra of these mutants in POPC/POPG membranes, the newly found signal that was attributed to POPG in the non-annular binding site was clearly absent, confirming the abolition of lipid binding in these sites (Fig. 7B, C).

The influence of specific lipid binding in KcsA in comparison to the chimeric potassium channel KcsA-Kv1.3, which contained 11 amino acids from the human Kv1.3 channel, was investigated by solid-state ${ }^{13} \mathrm{C}-{ }^{13} \mathrm{C}$ correlation NMR spectroscopy in asolection membranes [149], which contain the negatively charged phosphatidylinositol. The authors detected lipid crosspeaks in the region between 60 and 80 ppm (Fig. 7E), which represents the fingerprint region of lipid headgroups, indicative of tightly bound non-annular lipids. Remarkably, these crosspeaks were absent for KcsA-Kv1.3, which features a negatively charged aspartate at position 64 (Fig. 7F). This confirms the findings from ${ }^{31} \mathrm{P} N \mathrm{NMR}$, where a Arg to Leu mutation in position 64 also showed no lipid binding [183]. These results imply the tightly bound ${ }^{13} \mathrm{C}$ labeled lipids, most likely copurified from the $E$. coli inner membranes of the expression system for KcsA. It should be underlined that the asolectin lipids were not

${ }^{13} \mathrm{C}$ labeled, which suggests that the detected lipid-protein crosspeaks were indeed caused from non-annular lipids from the expression system. Very recently, the structural and functional role of the protein-lipid interface at the pore look region of the $\mathrm{K}^{+}$channel was investigated by solid-state NMR and molecular dynamics simulations, which support the view that the pore loop structure of the $\mathrm{K}^{+}$channel is stabilized by specific protein-lipid contacts [184].

\section{Perspective}

From the above examples, it may have become clear that solid-state NMR methods hold great potential for the investigation of lipid-protein interaction in membranes. Both the influence of proteins on the lipid matrix and direct interactions between lipids and proteins can be detected by the method with great precision. However, there are also some limitations of the technique. Some of which may possibly be overcome by further technical development but a few principle problems remain.

One problem arises from the fact that solid-state NMR samples of protein-membrane complexes usually represent multilamellar membrane preparations, where the protein is incorporated into the membrane or associated with its surface and multiple layers of such membranes are stacked on top of each other separated by a relatively thin water layer. In such an arrangement titration studies of either protein or a specific lipid to the preparation as can be done in solution NMR are not possible. In order to determine binding affinities between lipid and protein, separate samples containing varying amounts of either protein 
or the lipid of interest have to be prepared, which is somewhat tedious and consumes more material.

An other problem of the investigation of phospholipid-protein interaction in the membrane is the fact that phospholipid molecules have a relatively similar chemical structure and the spectroscopic distinction between different species is consequently difficult. This influences studies of the preferential interaction between a protein and a specific lipid species. Although unsaturated lipid chains provide unique NMR signals, these chains usually contain also 'normal' $\mathrm{CH}_{2}$ groups, which show exactly the same spectral characteristics as in saturated chains [185]. While specific deuteration of the lipid chains may help overcoming this complication, investigations have to be restricted to relatively simple lipid mixtures $[185,186]$. Although the lipid headgroups show some more chemical diversity, the ${ }^{31} \mathrm{P}$ chemical shift range of different lipid species is still restricted to about $3.5 \mathrm{ppm}$ [40], which often prevents the distinction of the lipid species in the presence of some line broadening accompanied by a decrease in resolution. Often, lipid-protein interactions in the membrane are of hydrophobic nature. This means, that only little alterations in the electrostatic environment of the nuclei occur and the concomitant chemical shift alterations are small and difficult to observe for broadened lipid signals [183].

Often, the most interesting lipid species that also play a functional role are present only in trace amounts in biological membranes. Although such lipid molecules would also feature unique NMR signals, like phosphoinositides or lipopolysaccharides $[187,188]$, due to the dynamic range of the receiver, the detection of such species is compromised. One solution to this problem could be ${ }^{13} \mathrm{C}$ labeling, which has been described for some phospholipids [189] or is commercially available for cholesterol. But ${ }^{31} \mathrm{P} N M R$, which seems to be the most logical approach to study phosphoinositides or lipopolysaccharides can not benefit as the ${ }^{31} \mathrm{P}$ nucleus already has a natural abundance of $100 \%$. ${ }^{2} \mathrm{H}$ NMR of specifically deuterated lipids can be used, but if lipid of interest is highly diluted, natural abundance ${ }^{2} \mathrm{H}$ of the nondeuterated lipids will start to play a role if the dilution exceeds a factor of $\sim 1000$.

A last point worth mentioning is the exchange between bulk lipids and annular sites, which occurs relatively rapidly on the NMR timescale $[10,183]$. Thus, magnetization transfer or dipolar coupling, which are key parameter that are measured by NMR, are weak and often very hard to observe in the liquid crystalline phase state of the membrane. Therefore, experiments that probe such quantities have to be carried out in the frozen state. Under these circumstances, fixed distances can be determined, but such numbers have to be discussed with great caution as the fluid state of the membrane is characterized by a high degree of conformational disorder and dynamics on the lipid side, which results in distributions rather than fixed distances [22,151]. As in NMR distances are measured via dipolar couplings or spin diffusion, the determined lengths are biased towards the shortest distance as dipolar couplings and spin diffusion rates scale by $r^{-3}$ or $r^{-6}$, respectively [38]. Except for tightly bound non-annular lipid molecules, protein-lipid interactions should be viewed as probability for contacts and interactions rather than fixed distances and quasicrystalline arrangements. This is all in agreement with a modern view of the biological membrane, which is characterized by a high degree of molecular dynamics, flexible and transient domain structure, and dynamic interaction patterns between lipids and proteins $[1,2,15,22]$. Solid-state NMR has significantly contributed to this current model of biological 
membranes and the multitude of molecular interactions of lipids and proteins in the fluid state.

\section{Acknowledgement}

I would like to thank Dr. Klaus Gawrisch for helpful discussions. Juliane Adler and Dr. Alexander Vogel are gratefully acknowledged for help with the preparation of Figs. 1 and 3. The work was supported by a grant from the Deutsche Forschungsgemeinschaft (SFB 1052, B6).

\section{Reference List}

1. S. H. White, A. S. Ladokhin, S. Jayasinghe, and K. Hristova, How membranes shape protein structure, J. Biol. Chem. 276 (2001) 32395-32398.

2. O. Soubias and K. Gawrisch, The role of the lipid matrix for structure and function of the GPCR rhodopsin, Biochim. Biophys. Acta. 1818 (2012) 234-240.

3. H. Khandelia, J. H. Ipsen, and O. G. Mouritsen, The impact of peptides on lipid membranes, Biochim. Biophys. Acta. 1778 (2008) 1528-1536.

4. M. F. Brown, Modulation of rhodopsin function by properties of the membrane bilayer, Chem. Phys. Lipids. 73 (1994) 159-180.

5. D. C. Mitchell, S. L. Niu, and B. J. Litman, Enhancement of G protein-coupled signaling by DHA phospholipids, Lipids. 38 (2003) 437-443.

6. S. J. Singer and G. L. Nicolson, The fluid mosaic model of the structure of cell membranes, Science 175 (1972) 720-731.

7. K. Simons and E. Ikonen, Functional rafts in cell membranes, Nature 387 (1997) 569-572.

8. M. J. Berridge and R. F. Irvine, Inositol phosphates and cell signalling, Nature. 341 (1989) 197-205.

9. R. F. Irvine, Inositol lipids in cell signalling, Curr. Opin. Cell Biol. 4 (1992) 212-219.

10. A. G. Lee, Lipid-protein interactions in biological membranes: a structural perspective, Biochim. Biophys. Acta. 1612 (2003) 1-40.

11. Y. Zhou, J. H. Morais-Cabral, A. Kaufman, and R. MacKinnon, Chemistry of ion coordination and hydration revealed by a $\mathrm{K}+$ channel-Fab complex at $2.0 \mathrm{~A}$ resolution, Nature 414 (2001) 43-48.

12. V. Cherezov, D. M. Rosenbaum, M. A. Hanson, S. G. F. Rasmussen, F. S. Thian, T. S. Kobilka, H. J. Choi, P. Kuhn, W. I. Weis, B. K. Kobilka, and R. C. Stevens, High-resolution crystal structure of an engineered human beta(2)-adrenergic $\mathrm{G}$ protein-coupled receptor, Science 318 (2007) 1258-1265.

13. P. J. Barrett, Y. Song, W. D. Van Horn, E. J. Hustedt, J. M. Schafer, A. Hadziselimovic, A. J. Beel, and C. R. Sanders, The amyloid precursor protein has a flexible transmembrane domain and binds cholesterol, Science. 336 (2012) 1168-1171. 
14. O. Soubias and K. Gawrisch, Rhodopsin-lipid interactions studied by NMR, Methods Enzymol. 522 (2013) 209-227.

15. M. F. Brown, Curvature forces in membrane lipid-protein interactions, Biochemistry. 51 (2012) 9782-9795.

16. J. A. Killian and T. K. Nyholm, Peptides in lipid bilayers: the power of simple models, Curr. Opin. Struct. Biol. 16 (2006) 473-479.

17. J. H. Davis, M. Auger, and R. S. Hodges, High resolution ${ }^{1} \mathrm{H}$ nuclear magnetic resonance of a transmembrane peptide, Biophys. J. 69 (1995) 1917-1932.

18. E. O. Stejskal and J. Schaefer, Carbon-13 nuclear magnetic resonance of polymers spinning at the magic angle, J. Am. Chem. Soc. 98 (1976) 1031-1032.

19. C. Tanford, The Hydrophobic Effect: Formation of Micelles and Biological Membranes, John Wiley \& Sons, New York, 1980.

20. M. Mihailescu, R. G. Vaswani, E. Jardon-Valadez, F. Castro-Roman, J. A. Freites, D. L. Worcester, A. R. Chamberlin, D. J. Tobias, and S. H. White, Acyl-chain methyl distributions of liquid-ordered and -disordered membranes, Biophys. J. 100 (2011) 1455-1462.

21. A. Bunge, P. Müller, M. Stöckl, A. Herrmann, and D. Huster, Characterization of the ternary mixture of sphingomyelin, POPC, and cholesterol: Support for an inhomogeneous lipid distribution at high temperatures, Biophys. J. 94 (2008) 2680-2690.

22. D. Huster, K. Arnold, and K. Gawrisch, Investigation of lipid organization in biological membranes by two-dimensional nuclear Overhauser enhancement spectroscopy, J. Phys. Chem. B 103 (1999) 243-251.

23. D. Huster and K. Gawrisch, NOESY NMR crosspeaks between lipid headgroups and hydrocarbon chains: spin diffusion or molecular disorder?, J. Am. Chem. Soc. 121 (1999) 1992-1993.

24. T. Bartels, R. S. Lankalapalli, R. Bittman, K. Beyer, and M. F. Brown, Raftlike mixtures of sphingomyelin and cholesterol investigated by solid-state ${ }^{2} \mathrm{H}$ NMR spectroscopy, J. Am. Chem. Soc. 130 (2008) 14521-14532.

25. S. E. Feller, D. Huster, and K. Gawrisch, Interpretation of NOESY cross-relaxation rates from molecular dynamics simulations of a lipid bilayer, J. Am. Chem. Soc. 121 (1999) 89638964.

26. L. S. Vermeer, B. L. de Groot, V. Reat, A. Milon, and J. Czaplicki, Acyl chain order parameter profiles in phospholipid bilayers: computation from molecular dynamics simulations and comparison with ${ }^{2}$ H NMR experiments, Eur. Biophys. J. 36 (2007) 919-931.

27. A. Vogel, K.-T. Tan, H. Waldmann, S. E. Feller, M. F. Brown, and D. Huster, Flexibility of ras lipid modifications studied by ${ }^{2} \mathrm{H}$ solid-state NMR and molecular dynamics simulations, Biophys. J. 93 (2007) 2697-2712.

28. T. P. Trouard, A. A. Nevzorov, T. M. Alam, C. Job, J. Zajicek, and M. F. Brown, Influence of cholesterol on dynamics of dimyristoylphosphatidylcholine bilayers as studied by deuterium NMR relaxation, J. Chem. Phys. 110 (1999) 8802-8818. 
29. A. A. Nevzorov, T. P. Trouard, and M. F. Brown, Lipid bilayer dynamics from simultaneous analysis of orientation and frequency dependence of deuterium spin-lattice and quadrupolar order relaxation, Phys. Rev. E. 58 (1998) 2259-2281.

30. H. A. Scheidt, D. Huster, and K. Gawrisch, Diffusion of cholesterol and its precursors in lipid membranes studied by ${ }^{1} \mathrm{H}$ pulsed field gradient magic angle spinning NMR, Biophys. J. 89 (2005) 2504-2512.

31. D. Huster, L. Xiao, and M. Hong, Solid-state NMR investigation of the dynamics of soluble and membrane-bound colicin la channel-forming domain, Biochemistry 40 (2001) 7662-7674.

32. R. S. Prosser and J. H. Davis, Dynamics of an integral membrane peptide: a deuterium NMR relaxation study of gramicidin, Biophys. J. 66 (1994) 1429-1440.

33. S. H. Park, F. Casagrande, B. B. Das, L. Albrecht, M. Chu, and S. J. Opella, Local and global dynamics of the G protein-coupled receptor CXCR1, Biochemistry. 50 (2011) 2371-2380.

34. M. Hong, X. Yao, K. S. Jakes, and D. Huster, Investigation of molecular motions by magicangle cross-polarization NMR spectroscopy, J. Phys. Chem. B 106 (2002) 7355-7364.

35. A. Vogel, G. Reuther, M. B. Roark, K. T. Tan, H. Waldmann, S. E. Feller, and D. Huster, Backbone conformational flexibility of the lipid modified membrane anchor of the human $\mathrm{N}$-Ras protein investigated by solid-state NMR and molecular dynamics simulation, Biochim. Biophys. Acta. 1798 (2010) 275-285.

36. G. Reuther, K.-T. Tan, A. Vogel, C. Nowak, J. Kuhlmann, H. Waldmann, and Huster D, The lipidated membrane anchor of the $\mathrm{N}$-ras protein shows an extensive dynamics as revealed by solid-state NMR, J. Am. Chem. Soc. 128 (2006) 13840-13846.

37. A. Watts, Solid-state NMR approaches for studying the interaction of peptides and proteins with membranes, Biochim. Biophys. Acta 1376 (1998) 297-318.

38. K. Schmidt-Rohr and H. W. Spiess, Multidimensional Solid-State NMR and Polymers, Academic Press, San Diego, 1994.

39. M. Mehring, Principles of High Resolution NMR in Solids, Springer Verlag, Heidelberg, 1983.

40. J. Schiller, M. Müller, B. Fuchs, K. Arnold, and D. Huster, ${ }^{31} \mathrm{P}$ NMR Spectroscopy of phospholipids: from micelles to membranes, Curr. Anal. Chem. 3 (2007) 283-301.

41. S. J. Opella and F. M. Marassi, Structure determination of membrane proteins by NMR spectroscopy, Chem. Rev. 104 (2004) 3587-3606.

42. B. Bechinger and E. S. Salnikov, The membrane interactions of antimicrobial peptides revealed by solid-state NMR spectroscopy, Chem. Phys. Lipids. 165 (2012) 282-301.

43. D. S. Wishart and B. D. Sykes, Chemical shifts as a tool for structure determination, Methods Enzymol. 239 (1994) 363-392.

44. E. R. Andrew, A. Bradbury, and R. G. Eades, Nuclear magnetic resonance spectra from a crystal rotated at high speed, Nature 182 (1958) 1659. 
45. M. M. Maricq and J. S. Waugh, NMR in rotating solids, J. Chem. Phys. 70 (1979) $3300-$ 3316.

46. A. Lakatos, K. Mors, and C. Glaubitz, How to investigate interactions between membrane proteins and ligands by solid-state NMR, Methods Mol. Biol. 914 (2012) 65-86.

47. D. Huster, Investigations of the structure and dynamics of membrane-associated peptides by magic angle spinning NMR, Prog. Nucl. Magn. Reson. Spectrosc. 46 (2005) 79107.

48. M. Renault, A. Cukkemane, and M. Baldus, Solid-state NMR spectroscopy on complex biomolecules, Angew. Chem. Int. Ed. 49 (2010) 8346-8357.

49. A. McDermott, Structure and dynamics of membrane proteins by magic angle spinning solid-state NMR, Annu. Rev. Biophys. 38 (2009) 385-403.

50. S. P. Brown, Applications of high-resolution ${ }^{1} \mathrm{H}$ solid-state NMR, Solid State Nucl. Magn Reson. 41 (2012) 1-27.

51. M. Hologne, V. Chevelkov, and B. Reif, Deuterated peptides and proteins in MAS solidstate NMR, Prog. Nucl. Magn. Reson. Spectrosc. 48 (2006) 211-232.

52. M. F. Brown, S. Lope-Piedrafita, G. V. Martinez, and H. I. Petrache, Solid-State Deuterium NMR Spectroscopy of Membranes, in: G.Webb (Ed.), Modern Magnetic Resonance, Springer, Heidelberg, 2006, pp.241-252.

53. J. H. Davis, The description of membrane lipid conformation, order and dynamics by ${ }^{2} \mathrm{H}$ NMR, Biochim. Biophys. Acta 737 (1983) 117-171.

54. J. Seelig, Deuterium magnetic resonance: theory and application to lipid membranes, Q. Rev. Biophys. 10 (1977) 353-418.

55. E. Strandberg, P. Tremouilhac, P. Wadhwani, and A. S. Ulrich, Synergistic transmembrane insertion of the heterodimeric PGLa/magainin 2 complex studied by solidstate NMR, Biochim. Biophys. Acta. 1788 (2009) 1667-1679.

56. M. F. Brown, M. P. Heyn, C. Job, S. Kim, S. Moltke, K. Nakanishi, A. A. Nevzorov, A. V. Struts, G. F. Salgado, and I. Wallat, Solid-state ${ }^{2} \mathrm{H}$ NMR spectroscopy of retinal proteins in aligned membranes, Biochim. Biophys. Acta. 1768 (2007) 2979-3000.

57. K. Gawrisch, O. Soubias, and M. Mihailescu, Insights from biophysical studies on the role of polyunsaturated fatty acids for function of G-protein coupled membrane receptors, Prostaglandins Leukot. Essent. Fatty Acids 79 (2008) 131-134.

58. J. Seelig, ${ }^{31} \mathrm{P}$ nuclear magnetic resonance and the head group structure of phospholipids in membranes, Biochim. Biophys. Acta 515 (1978) 105-140.

59. P. R. Cullis and B. de Kruijff, Lipid polymorphism and the functional roles of lipids in biological membranes, Biochim. Biophys. Acta 559 (1979) 399-420.

60. D. Huster, X. Yao, K. S. Jakes, and M. Hong, Conformational changes of colicin la channel-forming domain upon membrane binding: a solid-state NMR study, Biochim. Biophys. Acta 1561 (2002) 159-170. 
61. S. Schimmer, D. Lindner, P. Schmidt, A. G. Beck-Sickinger, D. Huster, and R. Rudolph, Functional characterization of the in vitro folded human $\mathrm{Y} 1$ receptor in lipid environment, Protein Pept. Lett. 17 (2010) 605-609.

62. A. Drechsler, G. Anderluh, R. S. Norton, and F. Separovic, Solid-state NMR study of membrane interactions of the pore-forming cytolysin, equinatoxin II, Biochim. Biophys. Acta. 1798 (2010) 244-251.

63. A. Vieler, H. A. Scheidt, P. Schmidt, C. Montag, J. F. Nowoisky, M. Lohr, C. Wilhelm, D. Huster, and R. Goss, The influence of phase transitions in phosphatidylethanolamine models on the activity of violaxanthin de-epoxidase, Biochim. Biophys. Acta. 1778 (2008) 1027-1034.

64. Y. Su, A. J. Waring, P. Ruchala, and M. Hong, Structures of beta-hairpin antimicrobial protegrin peptides in lipopolysaccharide membranes: mechanism of gram selectivity obtained from solid-state nuclear magnetic resonance, Biochemistry. 50 (2011) 2072-2083.

65. C. Muhle-Goll, S. Hoffmann, S. Afonin, S. L. Grage, A. A. Polyansky, D. Windisch, M. Zeitler, J. Burck, and A. S. Ulrich, Hydrophobic matching controls the tilt and stability of the dimeric platelet-derived growth factor receptor (PDGFR) beta transmembrane segment, J. Biol. Chem. 287 (2012) 26178-26186.

66. C. Kim, J. Spano, E. K. Park, and S. Wi, Evidence of pores and thinned lipid bilayers induced in oriented lipid membranes interacting with the antimicrobial peptides, magainin2 and aurein-3.3, Biochim. Biophys. Acta. 1788 (2009) 1482-1496.

67. E. S. Salnikov, H. Friedrich, X. Li, P. Bertani, S. Reissmann, C. Hertweck, J. D. O'Neil, J. Raap, and B. Bechinger, Structure and alignment of the membrane-associated peptaibols ampullosporin A and alamethicin by oriented $15 \mathrm{~N}$ and $31 \mathrm{P}$ solid-state NMR spectroscopy, Biophys. J. 96 (2009) 86-100.

68. Y. Su, W. F. DeGrado, and M. Hong, Orientation, dynamics, and lipid interaction of an antimicrobial arylamide investigated by ${ }^{19} \mathrm{~F}$ and ${ }^{31} \mathrm{P}$ solid-state NMR spectroscopy, J. Am. Chem. Soc. 132 (2010) 9197-9205.

69. K. Bertelsen, J. Dorosz, S. K. Hansen, N. C. Nielsen, and T. Vosegaard, Mechanisms of peptide-induced pore formation in lipid bilayers investigated by oriented ${ }^{31} \mathrm{P}$ solid-state NMR spectroscopy, PLoS. One. 7 (2012) e47745.

70. M. A. Sani, E. J. Dufourc, and G. Grobner, How does the Bax-alpha1 targeting sequence interact with mitochondrial membranes? The role of cardiolipin, Biochim. Biophys. Acta. 1788 (2009) 623-631.

71. D. K. Lee, J. R. Brender, M. F. Sciacca, J. Krishnamoorthy, C. Yu, and A. Ramamoorthy, Lipid Composition-Dependent Membrane Fragmentation and Pore-Forming Mechanisms of Membrane Disruption by Pexiganan (MSI-78), Biochemistry. 52 (2013) 3254-3262.

72. H. I. Petrache, S. W. Dodd, and M. F. Brown, Area per lipid and acyl length distributions in fluid phosphatidylcholines determined by ${ }^{2} \mathrm{H}$ NMR spectroscopy, Biophys. J. 79 (2000) 3172-3192. 
73. A. Vogel, C. P. Katzka, H. Waldmann, K. Arnold, M. F. Brown, and D. Huster, Lipid modifications of a ras peptide exhibit altered packing and mobility versus host membrane as detected by ${ }^{2} \mathrm{H}$ solid-state NMR, J. Am. Chem. Soc. 127 (2005) 12263-12272.

74. A. Leftin and M. F. Brown, An NMR database for simulations of membrane dynamics, Biochim. Biophys. Acta. 1808 (2011) 818-839.

75. H. A. Scheidt, T. Meyer, J. Nikolaus, D. J. Baek, I. Haralampiev, L. Thomas, R. Bittman, A. Herrmann, P. Müller, and Huster D., Cholesterol's Aliphatic Side Chain Structure Modulates Membrane Properties, Angew. Chem. Int. Ed. DOI: 10.1002/anie.201306753 (2013).

76. P. Barré, O. Zschörnig, K. Arnold, and D. Huster, Structural and dynamical changes of the bindin B18 peptide upon binding to lipid membranes. A solid-state NMR study, Biochemistry 42 (2003) 8377-8386.

77. M. F. Brown, R. L. Thurmond, S. W. Dodd, D. Otten, and K. Beyer, Composite membrane deformation on the mesoscopic length scale, Phys. Rev. E. 64 (2001) 010901.

78. G. V. Martinez, E. M. Dykstra, S. Lope-Piedrafita, C. Job, and M. F. Brown, NMR elastometry of fluid membranes in the mesoscopic regime, Phys. Rev. E. 66 (2002) 050902.

79. A. Vogel, T. Schröder, C. Lange, and D. Huster, Characterization of the myristoyl lipid modification of membrane-bound GCAP-2 by ${ }^{2} \mathrm{H}$ solid-state NMR spectroscopy, Biochim. Biophys. Acta 1768 (2007) 3171-3181.

80. A. Ramamoorthy, S. Thennarasu, D. K. Lee, A. Tan, and L. Maloy, Solid-state NMR investigation of the membrane-disrupting mechanism of antimicrobial peptides MSI-78 and MSI-594 derived from magainin 2 and melittin, Biophys. J. 91 (2006) 206-216.

81. H. A. Scheidt and D. Huster, Structure and dynamics of the myristoyl lipid modification of Src peptides determined by ${ }^{2} \mathrm{H}$ solid-state NMR spectroscopy, Biophys. J. 96 (2009) 36633672 .

82. M. Michalek, E. S. Salnikov, S. Werten, and B. Bechinger, Membrane interactions of the amphipathic amino terminus of huntingtin, Biochemistry. 52 (2013) 847-858.

83. G. Künze, P. Barre, H. A. Scheidt, L. Thomas, D. Eliezer, and D. Huster, Binding of the three-repeat domain of tau to phospholipid membranes induces an aggregated-like state of the protein, Biochim. Biophys. Acta. 1818 (2012) 2302-2313.

84. D. I. Fernandez, T. H. Lee, M. A. Sani, M. I. Aguilar, and F. Separovic, Proline facilitates membrane insertion of the antimicrobial peptide maculatin 1.1 via surface indentation and subsequent lipid disordering, Biophys. J. 104 (2013) 1495-1507.

85. X. Yu and G. A. Lorigan, Probing the Interaction of Arg9Cys Mutated Phospholamban with Phospholipid Bilayers by Solid-state NMR Spectroscopy, Biochim. Biophys. Acta. 1828 (2013) 2444-2449.

86. A. J. Mason, J. J. Lopez, M. Beyermann, and C. Glaubitz, A spectroscopic study of the membrane interaction of tuberoinfundibular peptide of 39 residues (TIP39), Biochim. Biophys. Acta 1714 (2005) 1-10. 
87. S. Thennarasu, R. Huang, D. K. Lee, P. Yang, L. Maloy, Z. Chen, and A. Ramamoorthy, Limiting an antimicrobial peptide to the lipid-water interface enhances its bacterial membrane selectivity: a case study of MSI-367, Biochemistry. 49 (2010) 10595-10605.

88. X. Yu and G. A. Lorigan, Probing the interaction of Arg9Cys mutated phospholamban with phospholipid bilayers by solid-state NMR spectroscopy, Biochim. Biophys. Acta. 1828 (2013) 2444-2449.

89. W. E. Teague, Jr., O. Soubias, H. Petrache, N. Fuller, K. G. Hines, R. P. Rand, and K. Gawrisch, Elastic properties of polyunsaturated phosphatidylethanolamines influence rhodopsin function, Faraday Discuss. 161 (2013) 383-395.

90. T. Huber, A. V. Botelho, K. Beyer, and M. F. Brown, Membrane model for the G-proteincoupled receptor rhodopsin: hydrophobic interface and dynamical structure, Biophys. J. 86 (2004) 2078-2100.

91. M. T. Eddy, T. C. Ong, L. Clark, O. Teijido, P. C. van der Wel, R. Garces, G. Wagner, T. K. Rostovtseva, and R. G. Griffin, Lipid dynamics and protein-lipid interactions in 2D crystals formed with the beta-barrel integral membrane protein VDAC1, J. Am. Chem. Soc. 134 (2012) 6375-6387.

92. M. Kobayashi, A. V. Struts, T. Fujiwara, M. F. Brown, and H. Akutsu, Fluid mechanical matching of $\mathrm{H}+$-ATP synthase subunit c-ring with lipid membranes revealed by $2 \mathrm{H}$ solidstate NMR, Biophys. J. 94 (2008) 4339-4347.

93. J. R. Brender, D. L. Heyl, S. Samisetti, S. A. Kotler, J. M. Osborne, R. R. Pesaru, and A. Ramamoorthy, Membrane disordering is not sufficient for membrane permeabilization by islet amyloid polypeptide: studies of IAPP(20-29) fragments, Phys. Chem. Chem. Phys. 15 (2013) 8908-8915.

94. A. Leftin, C. Job, K. Beyer, and M. F. Brown, Solid-State C NMR Reveals Annealing of Raft-Like Membranes Containing Cholesterol by the Intrinsically Disordered Protein alphaSynuclein, J. Mol. Biol. (2013) 10.

95. K. Mors, C. Roos, F. Scholz, J. Wachtveitl, V. Dotsch, F. Bernhard, and C. Glaubitz, Modified lipid and protein dynamics in nanodiscs, Biochim. Biophys. Acta. 1828 (2013) 1222-1229.

96. P. M. Macdonald, Q. Saleem, A. Lai, and H. H. Morales, NMR methods for measuring lateral diffusion in membranes, Chem. Phys. Lipids. 166 (2013) 31-44.

97. H. C. Gaede and K. Gawrisch, Lateral diffusion rates of lipid. water, and a hydrophobic drug in a multilamellar liposome, Biophys. J. 85 (2003) 1734-1740.

98. G. Oradd and G. Lindblom, Lateral diffusion studied by pulsed field gradient NMR on oriented lipid membranes, Magn Reson. Chem. 42 (2004) 123-131.

99. D. B. Fenske and H. C. Jarrell, Phosphorus-31 two-dimensional solid-state exchange NMR. Application to model membrane and biological systems, Biophys. J. 59 (1991) 55-69.

100. Q. Saleem, A. Lai, H. H. Morales, and P. M. Macdonald, Lateral diffusion of bilayer lipids measured via ${ }^{31}$ P CODEX NMR, Chem. Phys. Lipids. 165 (2012) 721-730. 
101. A. Pampel, J. Kärger, and D. Michel, Lateral diffusion of a transmembrane peptide in lipid bilayers studied by pulsed field gradient NMR in combination with magic angle sample spinning, Chem. Phys. Lett. 379 (2003) 555-561.

102. G. Oradd and G. Lindblom, NMR Studies of lipid lateral diffusion in the DMPC/gramicidin D/water system: peptide aggregation and obstruction effects, Biophys. J. 87 (2004) 980-987.

103. G. Oradd, A. Schmidtchen, and M. Malmsten, Effects of peptide hydrophobicity on its incorporation in phospholipid membranes--an NMR and ellipsometry study, Biochim. Biophys. Acta. 1808 (2011) 244-252.

104. P. Meier, E. Ohmes, and G. Kothe, Multipulse dynamic nuclear magnetic resonance of phospholipid membranes, J. Chem. Phys. 85 (1986) 3598-3614.

105. N. V. Eldho, S. E. Feller, S. Tristram-Nagle, I. V. Polozov, and K. Gawrisch, Polyunsaturated docosahexaenoic vs docosapentaenoic acid-differences in lipid matrix properties from the loss of one double bond, J. Am. Chem. Soc. 125 (2003) 6409-6421.

106. O. Soubias, I. V. Polozov, W. E. Teague, A. A. Yeliseev, and K. Gawrisch, Functional reconstitution of rhodopsin into tubular lipid bilayers supported by nanoporous media, Biochemistry. 45 (2006) 15583-15590.

107. O. Soubias, S. L. Niu, D. C. Mitchell, and K. Gawrisch, Lipid-rhodopsin hydrophobic mismatch alters rhodopsin helical content, J. Am. Chem. Soc. 130 (2008) 12465-12471.

108. K. Wüthrich, NMR of Proteins and Nucleic Acids, John Wiley \& Sons, New York, 1986.

109. J. Forbes, J. Bowers, X. Shan, L. Moran, E. Oldfield, and M. A. Moscarello, Some new developments in solid-state nuclear magnetic resonance spectroscopic studies of lipids and biological membranes, including the effect of cholesterol in model and natural systems, J. Chem. Soc. , Faraday Trans. 184 (1988) 3821-3849.

110. H. A. Scheidt and D. Huster, The interaction of small molecules with phospholipid membranes studied by ${ }^{1} \mathrm{H}$ NOESY NMR under magic-angle spinning, Acta Pharmacol. Sin. 29 (2008) 35-49.

111. C. Le Guerneve and M. Seigneuret, High-resolution mono- and multidimensional magic angle spinning ${ }^{1} \mathrm{H}$ nuclear magnetic resonance of membrane peptides in nondeuterated lipid membranes and $\mathrm{H}_{2} \mathrm{O}$, Biophys. J. 71 (1996) 2633-2644.

112. H. A. Scheidt, A. Pampel, L. Nissler, R. Gebhardt, and D. Huster, Investigation of the membrane localization and distribution of flavonoids by high-resolution magic angle spinning NMR spectroscopy, Biochim. Biophys. Acta 1663 (2004) 97-107.

113. N. Weizenmann, D. Huster, and H. A. Scheidt, Interaction of local anesthetics with lipid bilayers investigated by ${ }^{1} \mathrm{H}$ MAS NMR spectroscopy, Biochim. Biophys. Acta. 1818 (2012) 3010-3018.

114. A. Siarheyeva, J. J. Lopez, and C. Glaubitz, Localization of multidrug transporter substrates within model membranes, Biochemistry 45 (2006) 6203-6211. 
115. W. M. Yau, W. C. Wimley, K. Gawrisch, and S. H. White, The preference of tryptophan for membrane interfaces, Biochemistry 37 (1998) 14713-14718.

116. D. Aucoin, D. Camenares, X. Zhao, J. Jung, T. Sato, and S. O. Smith, High-resolution ${ }^{1} \mathrm{H}$ MAS RFDR NMR of biological membranes, J. Magn. Reson. 197 (2009) 77-86.

117. A. Jeziorna, T. Pawlak, K. Trzeciak-Karlikowska, P. Paluch, and M. J. Potrzebowski, Magic angle spinning NMR study of interaction of N-terminal sequence of dermorphin (Tyrd-Ala-Phe-Gly) with phospholipids, Biochim. Biophys. Acta. 1818 (2012) 2579-2587.

118. A. Ramamoorthy and J. Xu, 2D ${ }^{1} \mathrm{H} /{ }^{1} \mathrm{H}$ RFDR and NOESY NMR experiments on a membrane-bound antimicrobial peptide under magic angle spinning, J. Phys. Chem. B 117 (2013) 6693-6700.

119. D. Huster, K. Kuhn, D. Kadereit, H. Waldmann, and K. Arnold, High resolution magic angle spinning NMR for the investigation of a ras lipopeptide in a lipid bilayer, Angew. Chem. Int. Ed. 40 (2001) 1056-1058.

120. D. Huster, A. Vogel, C. Katzka, H. A. Scheidt, H. Binder, S. Dante, T. Gutberlet, O. Zschörnig, H. Waldmann, and K. Arnold, Membrane insertion of a lipidated ras peptide studied by FTIR, solid-state NMR, and neutron diffraction spectroscopy, J. Am. Chem. Soc. 125 (2003) 4070-4079.

121. D. Huster, X. Yao, and M. Hong, Membrane protein topology probed by ${ }^{1} \mathrm{H}$ spin diffusion from lipids using solid-state NMR spectroscopy, J. Am. Chem. Soc. 124 (2002) 874883.

122. M. Hong and K. Schmidt-Rohr, Magic-angle spinning NMR techniques for measuring long-range distances in biological macromolecules, Acc. Chem. Res. 46 (2013) 2154-2163.

123. R. Mani, S. D. Cady, M. Tang, A. J. Waring, R. I. Lehrer, and M. Hong, Membranedependent oligomeric structure and pore formation of a beta-hairpin antimicrobial peptide in lipid bilayers from solid-state NMR, Proc. Natl. Acad. Sci. U. S. A. 103 (2006) 1624216247.

124. M. Hong, Structure, topology, and dynamics of membrane peptides and proteins from solid-state NMR Spectroscopy, J. Phys. Chem. B 111 (2007) 10340-10351.

125. H. Yao and M. Hong, Membrane-dependent conformation, dynamics, and lipid interactions of the fusion peptide of the paramyxovirus PIV5 from solid-state NMR, J. Mol. Biol. 425 (2013) 563-576.

126. P. Agrawal, S. Kiihne, J. Hollander, D. Langosch, and Groot $\mathrm{H}$. de, ${ }^{13} \mathrm{C}$ and ${ }^{15} \mathrm{~N} N M R$ evidence for peripheral intercalation of uniformly labeled fusogenic peptides incorporated in a biomimetic membrane, Biochim. Biophys. Acta. 1768 (2007) 3020-3028.

127. S. Theisgen, H. A. Scheidt, A. Magalhaes, T. J. Bonagamba, and D. Huster, A solid-state NMR study of the structure and dynamics of the myristoylated N-terminus of the guanylate cyclase-activating protein-2, Biochim. Biophys. Acta. 1798 (2010) 266-274. 
128. A. Kijac, A. Y. Shih, A. J. Nieuwkoop, K. Schulten, S. G. Sligar, and C. M. Rienstra, Lipidprotein correlations in nanoscale phospholipid bilayers determined by solid-state nuclear magnetic resonance, Biochemistry. 49 (2010) 9190-9198.

129. D. Krepkiy, K. Gawrisch, and K. J. Swartz, Structural interactions between lipids, water and S1-S4 voltage-sensing domains, J. Mol. Biol. 423 (2012) 632-647.

130. B. Meyer and T. Peters, NMR Spectroscopy techniques for screening and identifying ligand binding to protein receptors, Angew. Chem. Int. Ed. 42 (2003) 864-890.

131. O. Soubias and K. Gawrisch, Probing specific lipid-protein interaction by saturation transfer difference NMR spectroscopy, J. Am. Chem. Soc. 127 (2005) 13110-13111.

132. O. Soubias, W. E. Teague, and K. Gawrisch, Evidence for specificity in lipid-rhodopsin interactions, J. Biol. Chem. 281 (2006) 33233-33241.

133. R. G. Griffin, Dipolar recoupling in MAS spectra of biological solids, Nat. Struct. Biol. 5 (1998) 508-512.

134. S. Dusold and A. Sebald, Dipolar recoupling under magic-angle spinning conditions, Ann. Rep. NMR Spectrosc. 41 (2000) 185-264.

135. J. S. Waugh, Uncoupling of local field spectra in nuclear magnetic resonance: determination of atomic positions in solids, Proc. Natl. Acad. Sci. U. S. A. 73 (1976) 13941397.

136. D. P. Raleigh, F. Creuzet, S. K. Das Gupta, M. H. Levitt, and R. G. Griffin, Measurement of internuclear distances in polycristalline solids: rotationally enhanced transfer of nuclear spin magnetization, J. Am. Chem. Soc. 111 (1989) 4502-4503.

137. T. Gullion and J. Schaefer, Detection of Weak Heteronuclear Dipolar Couplings by Rotational-Echo Double-Resonance Nuclear Magnetic Resonance, in: W.S.Warren (Ed.), Advances in magnetic resonance, Academic Press, San Diego, 1989, pp.57-83.

138. K. Schmidt-Rohr and M. Hong, Measurements of carbon to amide-proton distances by C-H dipolar recoupling with N-15 NMR detection, J. Am. Chem. Soc. 125 (2003) 5648-5649.

139. E. Harada, Y. Todokoro, H. Akutsu, and T. Fujiwara, Detection of peptide-phospholipid interaction sites in bilayer membranes by ${ }^{13} \mathrm{C}$ NMR spectroscopy: observation of ${ }^{2} \mathrm{H} /{ }^{31} \mathrm{P}$ selective ${ }^{1} \mathrm{H}$-depolarization under magic-angle spinning, J. Am. Chem. Soc. 128 (2006) 10654-10655.

140. M. Tang, A. J. Waring, R. I. Lehrer, and M. Hong, Effects of guanidinium-phosphate hydrogen bonding on the membrane-bound structure and activity of an arginine-rich membrane peptide from solid-state NMR spectroscopy, Angew. Chem. Int. Ed. 47 (2008) 3202-3205.

141. M. Tang, A. J. Waring, and M. Hong, Effects of arginine density on the membranebound structure of a cationic antimicrobial peptide from solid-state NMR, Biochim. Biophys. Acta. 1788 (2009) 514-521. 
142. O. Toke, W. L. Maloy, S. J. Kim, J. Blazyk, and J. Schaefer, Secondary structure and lipid contact of a peptide antibiotic in phospholipid bilayers by REDOR, Biophys. J. 87 (2004) 662674.

143. A. Tsutsumi, N. Javkhlantugs, A. Kira, M. Umeyama, I. Kawamura, K. Nishimura, K. Ueda, and A. Naito, Structure and orientation of bovine lactoferrampin in the mimetic bacterial membrane as revealed by solid-state NMR and molecular dynamics simulation, Biophys. J. 103 (2012) 1735-1743.

144. C. M. Gabrys, W. Qiang, Y. Sun, L. Xie, S. D. Schmick, and D. P. Weliky, Solid-state nuclear magnetic resonance measurements of HIV fusion peptide CO to Lipid P proximities support similar partially inserted membrane locations of the alpha helical and beta sheet peptide structures, J. Phys. Chem. A. (2013).

145. Y. H. Lam, A. Hung, R. S. Norton, F. Separovic, and A. Watts, Solid-state NMR and simulation studies of equinatoxin II N-terminus interaction with lipid bilayers, Proteins. 78 (2010) 858-872.

146. Y. Su, T. Doherty, A. J. Waring, P. Ruchala, and M. Hong, Roles of arginine and lysine residues in the translocation of a cell-penetrating peptide from ${ }^{13} \mathrm{C},{ }^{31} \mathrm{P}$, and ${ }^{19} \mathrm{~F}$ solid-state NMR, Biochemistry. 48 (2009) 4587-4595.

147. N. M. Szeverenyi, M. J. Sullivan, and G. E. Maciel, Observation of spin exchange by twodimensional Fourier transform ${ }^{13} \mathrm{C}$ cross polarization magic-angle spinning, J. Magn Reson. 47 (1982) 462-475.

148. V. S. Mithu, S. Bakthavatsalam, and P. K. Madhu, ${ }^{13} \mathrm{C}-{ }^{13} \mathrm{C}$ homonuclear recoupling in solid-state nuclear magnetic resonance at a moderately high magic-angle-spinning frequency, PLoS. One. 8 (2013) e50504.

149. M. Weingarth, A. Prokofyev, E. A. van der Cruijsen, D. Nand, A. M. Bonvin, O. Pongs, and M. Baldus, Structural determinants of specific lipid binding to potassium channels, J. Am. Chem. Soc. 135 (2013) 3983-3988.

150. G. M. Clore and J. Iwahara, Theory, practice, and applications of paramagnetic relaxation enhancement for the characterization of transient low-population states of biological macromolecules and their complexes, Chem. Rev. 109 (2009) 4108-4139.

151. A. Vogel, H. A. Scheidt, and D. Huster, The distribution of lipid attached EPR probes in bilayers. Application to membrane protein topology, Biophys. J. 85 (2003) 1691-1701.

152. D. Huster, P. Müller, K. Arnold, and A. Herrmann, Dynamics of membrane penetration of the fluorescent 7-nitrobenz-2-oxa-1,3-diazol-4-yl (NBD) group attached to an acyl chain of phosphatidylcholine, Biophys. J. 80 (2001) 822-831.

153. H. A. Scheidt, P. Müller, A. Herrmann, and D. Huster, The potential of fluorescent and spin-labeled steroid analogs to mimic natural cholesterol, J. Biol. Chem. 278 (2003) 4556345569.

154. A. Kyrychenko and A. S. Ladokhin, Molecular dynamics simulations of depth distribution of spin-labeled phospholipids within lipid bilayer, J. Phys. Chem. B. 117 (2013) 5875-5885. 
155. A. Kurz, A. Bunge, A.-K. Windeck, M. Rost, W. Flasche, A. Arbuzova, D. Strobach, S. Müller, J. Liebscher, D. Huster, and A. Herrmann, Lipid-anchored oligonucleotides for stable double-helix formation in distinct membrane domains, Angew. Chem. Int. Ed Engl. 45 (2006) 4440-4444.

156. J. A. Butterwick and R. MacKinnon, Solution structure and phospholipid interactions of the isolated voltage-sensor domain from KvAP, J. Mol. Biol. 403 (2010) 591-606.

157. L. Thomas, H. A. Scheidt, A. Bettio, D. Huster, A. G. Beck-Sickinger, K. Arnold, and O. Zschornig, Membrane interaction of neuropeptide $Y$ detected by EPR and NMR spectroscopy, Biochim. Biophys. Acta 1714 (2005) 103-113.

158. L. Thomas, H. A. Scheidt, A. Bettio, A. G. Beck-Sickinger, D. Huster, and O. Zschornig, The interaction of neuropeptide $Y$ with negatively charged and zwitterionic phospholipid membranes, Eur. Biophys. J. 38 (2009) 663-677.

159. K. Wagner, A. G. Beck-Sickinger, and D. Huster, Structural investigations of a human calcitonin-derived carrier peptide in membrane environment by solid-state NMR, Biochemistry 43 (2004) 12459-81246.

160. L. Brunsveld, H. Waldmann, and D. Huster, Membrane binding of lipidated Ras peptides and proteins - The structural point of view, Biochim. Biophys. Acta 1788 (2009) 273-288.

161. G. Reuther, K.-T. Tan, J. Köhler, C. Nowak, A. Pampel, K. Arnold, J. Kuhlmann, H. Waldmann, and Huster D., Structural model of the membrane-bound C terminus of lipidmodified human N-ras protein, Angew. Chem. Int. Ed. 45 (2006) 5387-5390.

162. A. Vogel, G. Reuther, K. Weise, G. Triola, J. Nikolaus, K. T. Tan, C. Nowak, A. Herrmann, H. Waldmann, R. Winter, and D. Huster, The lipid modifications of Ras that sense membrane environments and induce local enrichment, Angew. Chem. Int. Ed. 48 (2009) 8784-8787.

163. O. Rocks, A. Peyker, M. Kahms, P. J. Verveer, C. Koerner, M. Lumbierres, J. Kuhlmann, H. Waldmann, A. Wittinghofer, and P. I. H. Bastiaens, An acylation cycle regulates localization and activity of palmitoylated Ras isoforms, Science 307 (2005) 1746-1752.

164. S. J. Plowman and J. F. Hancock, Ras signaling from plasma membrane and endomembrane microdomains, Biochim. Biophys. Acta 1746 (2005) 274-283.

165. M. Hong and Y. Su, Structure and dynamics of cationic membrane peptides and proteins: insights from solid-state NMR, Protein Sci. 20 (2011) 641-655.

166. S. Bhattacharjya and A. Ramamoorthy, Multifunctional host defense peptides: functional and mechanistic insights from NMR structures of potent antimicrobial peptides, FEBS J. 276 (2009) 6465-6473.

167. F. Porcelli, A. Ramamoorthy, G. Barany, and G. Veglia, On the role of NMR spectroscopy for characterization of antimicrobial peptides, Methods Mol. Biol. 1063 (2013) 159-180. 
168. R. E. Hancock and R. Lehrer, Cationic peptides: a new source of antibiotics, Trends Biotechnol. 16 (1998) 82-88.

169. M. Tang, A. J. Waring, and M. Hong, Phosphate-mediated arginine insertion into lipid membranes and pore formation by a cationic membrane peptide from solid-state NMR, J. Am. Chem. Soc. 129 (2007) 11438-11446.

170. W. C. Wimley and S. H. White, Experimentally determined hydrophobicity scale for proteins at membrane interfaces, Nat. Struct. Biol. 3 (1996) 842-848.

171. T. Hessa, H. Kim, K. Bihlmaier, C. Lundin, J. Boekel, H. Andersson, I. Nilsson, S. H. White, and Heijne $\mathrm{G}$. von, Recognition of transmembrane helices by the endoplasmic reticulum translocon, Nature. 433 (2005) 377-381.

172. P. A. Marasinghe, J. J. Buffy, K. Schmidt-Rohr, and M. Hong, Membrane curvature change induced by an antimicrobial peptide detected by ${ }^{31} \mathrm{P}$ exchange NMR, J. Phys. Chem. B. 109 (2005) 22036-22044.

173. R. Mani, A. J. Waring, R. I. Lehrer, and M. Hong, Membrane-disruptive abilities of betahairpin antimicrobial peptides correlate with conformation and activity: a ${ }^{31} \mathrm{P}$ and ${ }^{1} \mathrm{H} N M R$ study, Biochim. Biophys. Acta. 1716 (2005) 11-18.

174. R. Mani, J. J. Buffy, A. J. Waring, R. I. Lehrer, and M. Hong, Solid-state NMR investigation of the selective disruption of lipid membranes by protegrin-1, Biochemistry. 43 (2004) 13839-13848.

175. K. Palczewski, T. Kumasaka, T. Hori, C. A. Behnke, H. Motoshima, B. A. Fox, Trong Le, I, D. C. Teller, T. Okada, R. E. Stenkamp, M. Yamamoto, and M. Miyano, Crystal structure of rhodopsin: A G protein-coupled receptor, Science 289 (2000) 739-745.

176. H. W. Choe, Y. J. Kim, J. H. Park, T. Morizumi, E. F. Pai, N. Krauss, K. P. Hofmann, P. Scheerer, and O. P. Ernst, Crystal structure of metarhodopsin II, Nature. 471 (2011) 651655.

177. V. Katritch, V. Cherezov, and R. C. Stevens, Structure-function of the G protein-coupled receptor superfamily, Annu. Rev. Pharmacol. Toxicol. 53 (2013) 531-556.

178. D. Salom, D. T. Lodowski, R. E. Stenkamp, Trong Le, I, M. Golczak, B. Jastrzebska, T. Harris, J. A. Ballesteros, and K. Palczewski, Crystal structure of a photoactivated deprotonated intermediate of rhodopsin, Proc. Natl. Acad. Sci. U. S. A. 103 (2006) 1612316128.

179. A. V. Botelho, N. J. Gibson, R. L. Thurmond, Y. Wang, and M. F. Brown, Conformational energetics of rhodopsin modulated by nonlamellar-forming lipids, Biochemistry. 41 (2002) 6354-6368.

180. O. Soubias, W. E. Teague, Jr., K. G. Hines, D. C. Mitchell, and K. Gawrisch, Contribution of membrane elastic energy to rhodopsin function, Biophys. J. 99 (2010) 817-824.

181. H. Schrempf, O. Schmidt, R. Kummerlen, S. Hinnah, D. Muller, M. Betzler, T. Steinkamp, and R. Wagner, A prokaryotic potassium ion channel with two predicted transmembrane segments from Streptomyces lividans, EMBO J. 14 (1995) 5170-5178. 
182. F. I. Valiyaveetil, Y. Zhou, and R. MacKinnon, Lipids in the structure, folding, and function of the KcsA K+ channel, Biochemistry. 41 (2002) 10771-10777.

183. P. Marius, M. R. de Planque, and P. T. Williamson, Probing the interaction of lipids with the non-annular binding sites of the potassium channel KcsA by magic-angle spinning NMR, Biochim. Biophys. Acta. 1818 (2012) 90-96.

184. E. A. van der Cruijsen, D. Nand, M. Weingarth, A. Prokofyev, S. Hornig, A. A. Cukkemane, A. M. Bonvin, S. Becker, R. E. Hulse, E. Perozo, O. Pongs, and M. Baldus, Importance of lipid-pore loop interface for potassium channel structure and function, Proc. Natl. Acad. Sci. U. S. A. 110 (2013) 13008-13013.

185. D. Huster, K. Arnold, and K. Gawrisch, Influence of docosahexaenoic acid and cholesterol on lateral lipid organization in phospholipid membranes, Biochemistry 37 (1998) 17299-17308.

186. D. Huster, K. Arnold, and K. Gawrisch, Strength of $\mathrm{Ca}^{2+}$ binding to retinal lipid membranes: consequences for lipid organization., Biophys. J. 78 (2000) 3011-3018.

187. S. Mitra, C. A. Traughber, M. K. Brannon, S. Gomez, and D. G. Capelluto, Ubiquitin Interacts with the Tollip C2 and CUE Domains and Inhibits Binding of Tollip to Phosphoinositides, J. Biol. Chem. 288 (2013) 25780-25791.

188. F. Ciesielski, D. C. Griffin, M. Rittig, I. Moriyon, and B. B. Bonev, Interactions of lipopolysaccharide with lipid membranes, raft models - a solid state NMR study, Biochim. Biophys. Acta. 1828 (2013) 1731-1742.

189. J. M. Boettcher, R. L. vis-Harrison, M. C. Clay, A. J. Nieuwkoop, Y. Z. Ohkubo, E. Tajkhorshid, J. H. Morrissey, and C. M. Rienstra, Atomic view of calcium-induced clustering of phosphatidylserine in mixed lipid bilayers, Biochemistry. 50 (2011) 2264-2273. 